\title{
Extensions of the Constructivist Real Number System
}

\author{
E. E. Escultura \\ GVP-Professor V. Lakshmikantham Institute for Advanced Studies, GVP College of Engineering, JNT University, \\ Kakinada, India \\ Email: escultur36@gmail.com
}

How to cite this paper: Escultura, E.E. (2018) Extensions of the Constructivist Real Number System. Advances in Pure Mathematics, 8, 720-754.

https://doi.org/10.4236/apm.2018.88044

Received: February 8, 2018

Accepted: August 17, 2018

Published: August 20, 2018

Copyright (c) 2018 by author and Scientific Research Publishing Inc. This work is licensed under the Creative Commons Attribution International License (CC BY 4.0).

http://creativecommons.org/licenses/by/4.0/

\begin{abstract}
The paper reviews the most consequential defects and rectification of traditional mathematics and its foundations. While this work is only the tip of the iceberg, so to speak, it gives us a totally different picture of mathematics from what we have known for a long time. This journey started with two teasers posted in SciMath in 1997: 1 ) The equation $1=0.99 \ldots$ does not make sense. 2 ) The concept $i=\sqrt{-1}$ does not exist. The first statement sparked a debate that raged over a decade. Both statements generated a series of publications that continues to grow to this day. Among the new findings are: 3 ) There does not exist nondenumerable set. 4) There does not exist non-measurable set. 5) Cantor's diagonal method is flawed. 6) The real numbers are discrete and countable. 7) Formal logic does not apply to mathematics. The unfinished debate between logicism, intuitionism-constructivism and formalism is resolved. The resolution is the constructivist foundations of mathematics with a summary of all the rectification undertaken in 2015, 2016 and in this paper. The extensions of the constructivist real number system include the complex vector plane and transcendental functions. Two important results in the 2015 are noted: The solution and resolution of Hilbert's 23 problems that includes the resolution of Fermat's last theorem and proof Goldbach's conjecture.
\end{abstract}

\section{Keywords}

Constructivism, Dark Number, Fermat's Conjecture, g-Norm, g-Sequence, g-Limit, Goldbach's Conjecture, Truncation, Vector Operators $j$ and $h_{\theta}$

\section{Introduction}

Four previous papers, delineated the boundary of region of validity of the real number system $\mathbf{R}$ and its foundations and established $\mathbf{R}$ on the terminating de- 
cimals [1] [2] [3] [4]. The extension of $\mathbf{R}$ to its boundary, introduction of new concepts, re-definition of previously ill-defined concepts and imposition of new requirements to avoid ambiguity, errors and paradoxes (contradictions) extended the domain of $\mathbf{R}$ to the nonterminating decimals and established a new mathematical space called the constructivist real number system $\mathbf{R}^{\star}$ [5]. Even $\mathbf{R}^{\star}$ has a lot to be desired. But the extension of the definition of exponent, logarithm and exponential and logarithmic functions to nonterminating decimals as well as the introduction of the vector operators $\mathbf{j}$ and $\mathbf{h}_{\varphi}$ effectively extends $\mathbf{R}^{\star}$ to transcendental functions and the complex vector plane.

We provide a summary of the great debate of the $20^{\text {th }}$ century between the three philosophies or schools of thought of mathematics, namely, logicism, intuitionism-constructivism and formalism represented by Bertrand Russell [6], L. E. J. Brouwer [7] and David Hilbert [8], respectively. Logicism attempted to build mathematics on symbolic logic. The issue: which one provides firm foundations for mathematics? None of them won the debate but we identify their main contributions and add our own to resolve the debate and call the resolution the constructivist foundations of mathematics. The debate and its resolution comprise the core of this paper. The debate started when Russell sent a letter to Gottlob Frege [9] confounding him with the Russell Antimony (Russell's contribution) [10]:

Let $M$ be the set of all sets where each element does not belong to itself, i.e., $M=\{m: m \notin m\}$. Either $M \in M$ or $M \notin M$. If $M \notin M$, its defining conditions hold; therefore $M \in M$. On the other hand, if $M \in M$, then $M$ also satisfies its defining condition; therefore $M \in M$ and $M \notin M$.

The Russell Antimony is also called the Law of Excluded Middle which is the basis of the indirect proof. Its rejection by Brouwer gave rise to intuitionism-constructivism, Brower's contribution. Brouwer rejected his earlier contribution-the fixed-point theorem-which was proved with the indirect proof [11]. Hilbert recognized that the concepts of individual thought are inaccessible to others and cannot be the subject matter of mathematics. His remedy: represent the concepts of individual thought by objects in the real world such as words, letters and figures subject to consistent basic premises or axioms. The axioms are neither true nor false but mathematical arguments, conclusions and proofs of theorems are based on and consistent with them. Any statement that contradicts any of the axioms or their conclusions is called a counterexample; it dismisses and proves the statement false. In other words, mathematics is the study of symbols and other objects subject to these requirements. The axioms define a mathematical space such as algebra, analysis and geometry. The choice of the axioms is arbitrary depending on what the mathematical space is intended for. Consequently, it is possible to construct independent contradictory mathematical spaces such as Abelean and non-Abelean groups and Euclidean and non-Euclidean geometries. Scientists use mathematics as the language of science because of its precision and many mathematical spaces come from and meet the 
needs of science. Clearly, symbolic logic is useless in mathematics because it has nothing to do with the axioms. Mathematics has its own logic that we call rational thought. The precision of mathematics lies not in computation and measurement because they are its most imprecise aspects but in the way it establishes conclusions and creates new concepts.

\section{Mathematical Space}

A mathematical space consists of a set of concepts, binary or other operations and relations subject to consistent basic axioms. Ernst Zermelo and Abraham Fränkel attempted to construct set theory [12] as a mathematical space but they failed because one of the field axioms [13], the axiom of choice [14], is false on infinite set due to its inherent ambiguity [1]. Consequently, the attempt of logicism to develop set theory as the universal language of mathematics did not materialize. Therefore, we use mainly the concepts of naïve set theory not its results but identify its defects at the same time.

\subsection{Abstract and Physical Concepts}

Both abstract and physical concepts are created by individual thought. The difference: although both of them are represented by objects in the real world such as word, symbol, number and figure, an abstract concept has no referent in the real world while a physical concept refers to an object in the real world that everyone can look at and examine. Examples of abstract concept: time, distance and dimension; we cannot find them in the real world. The concept time is invented by thought to express a relation between events that tells us which of them occurred first. Distance is a relation between objects in the real world that describes their relative positions. For example, the objects in the sky called Milky Way and Andromeda are physical concepts; they are the physical referents of the physical concepts "Milky Way" and "Andromeda". The distance between them can be measured and computed. Some physical concepts like the superstring, fundamental building block of matter, is not directly observable. They were discovered only indirectly through their impact in the real world by qualitative mathematics, the mathematical model of rational thought [15].

Lack of distinction between abstract and physical concepts can lead to erroneous science. For example, Albert Einstein considered time a physical concept. He got the twin paradox, a contradiction. Of course, thought can create nonsense. Examples: a bag half its size or the snake that swallowed itself. But it is also capable of correcting them. However, uncorrected error can be tragic as the disastrous final flight of the Columbia Space Shuttle showed [16].

\subsection{Ambiguity, Errors and Paradoxes (Contradictions)}

The undefined concepts or terms of traditional mathematics are examples of ambiguous concepts. They include vacuous and undefined concepts. An example of undefined concept is a nonterminating decimal. An example of vacuous 
concept is "the root of the equation $x^{2}+1=0$ ", denoted by $i=\sqrt{-1}$. This concept is vacuous because the unary operator $\sqrt{x}$ is defined only when $x \geq 0$ and a perfect square and -1 is not. Consequently,

$$
i=\sqrt{-1}=\sqrt{\frac{1}{-1}}=-i
$$

from which follows that

$$
i=0 \text { and } 1=0
$$

which collapses both the real and complex number systems.

Another vacuous concept is "the greatest integer". Suppose we want to find the greatest integer. Let $N=$ the greatest integer. By the trichotomy axiom, one and only one of the following holds: $N<1, N=1, N>1$. The left inequality is obviously false. It follows from the right inequality that $N^{\mathcal{Z}}>N$ which contradicts our assumption that $N$ is the greatest integer. Therefore, $N=1$, a contradiction. This is called the Perron paradox [17].

There are two sources of this paradox;

- The trichotomy axiom is false in the real number system [5] but it is true and follows from the lexicographic ordering of the constructivist real number system $\mathbf{R}^{\star}$ [5].

- To avoid error constructivism requires proof of existence of solution of a problem before solving it. This is a common error, especially, in differential equations where a solution is assumed without first proving that a solution exists (e.g., let $f(x)$ be the solution ...). Such "solution" if found need not be a solution.

\subsection{Ambiguity of the Concept "Irrational"}

Since the binary operations addition and multiplication are defined only on terminating decimals the nonterminating decimals are ill-defined and, therefore, ambiguous in the real number system. Therefore, any concept defined in terms of nonterminating decimals is ambiguous, ill-defined. An example of such ambiguous concept is irrational number, i.e., nonperiodic nonterminating decimal. Furthermore, periodicity or non-periodicity of a nonterminating decimal is not verifiable because verification is an endless process. Thus, an irrational number has at least two layers of ambiguity-being ill-defined and having infinite decimal digits. This ambiguity is illustrated by the fact that the sum $\sqrt{3}$ and $\sqrt{2}$ cannot be computed. Moreover, not every rational number is the quotient of two integers; only when the divisor has no prime factor other than 2 and 5. For example, $2 / 3=1.66 \ldots$ Thus, the rationals coincide with the terminating decimals and they are the only defined real numbers.

\subsection{Infinity}

In traditional mathematics a set is infinite if it can be put into one-one-correspondence with a proper subset. We modify this definition by 
identifying the concept infinity with its essential property of inexhaustibility. We take inexhaustibility as the defining quality of infinity and this clearly includes the traditional definition. For example, if we count the digits of a nonterminating decimal and label the digits we have already counted by, say, $x_{1}, x_{2}, \cdots, x_{n}$, then a sequence, $x_{1}, x_{2}, \cdots, x_{n}, n=1,2, \cdots$ is generated that has no last element and the counting is never complete. This is an ambiguity. It is not the case with a terminating decimal where there is a last element so that its digits are finite. This is an example of countable infinity denoted by $\infty$. It is a concept that pervades mathematics and the only type of infinity that exists as we shall see later. It is neither a real number nor a counting number and, naturally, the binary additive and multiplicative operations do not apply to it and if real numbers are added to a given real number one at a time $\infty$ can never be reached. Thus, there is no boundary between the real numbers and infinity that can be crossed.

\subsection{The Universal and Existential Quantifier}

Let $S$ be nonempty set and suppose we want to prove that "every element of $S$ has property P". Start with an element $x_{1}$ and suppose it has this property (otherwise, the statement is outright false), then take another element $x_{2}$ and check if it has this property, etc. Then since $S$ is inexhaustible verification of the truth of this statement is never complete, i.e., the statement is ambiguous. Similarly, by the same algorithm but starting with an element that does not have this property, it may not be possible to prove that there exists an element of $S$ that has this property which is an ambiguity. What this all means is that the application of the universal or existential quantifier to infinite set brings in ambiguity to a mathematical space. Every infinite mathematical space is presently tainted with this type of ambiguity from the definition of limit of real analysis through the field axioms of the real number system [13]. Thus, the real number system is presently ambiguous.

\subsection{Other Defects of the Real Number System and Its Foundations}

We enumerate the other defects of the real number system that have some bearing on this paper:

- The trichotomy axiom, one of the field axioms of the real number system, is false; a counterexample to it is constructed in [5].

- The axiom of choice, one of the axioms of the real number system, is false on infinite set [1].

- Only the terminating decimals, which coincide with the rational numbers, are defined in the real number system; in particular, the nonterminating decimals are ill-defined [5].

- The power set of a set leads to the Russell antimony; therefore, like the universal set, it does not exist [1].

- Cantor's diagonal method generates only a countably infinite set; since the union of countably infinite sets is countable, the real numbers are countably 
infinite.

- Since the cardinality of a set is defined by the power set and the power set of the countably infinite set does not exist then the only cardinality that exists is countably infinite [1].

- Reference [13] exhibits a non-measurable set. However, the proof uses the axiom of choice. Therefore, as of this time, there is no non-measurable set.

- Since $\sqrt[n]{x}$ is defined only when $x$ is a perfect nth power then the fractional root of $x$ does not exist unless $x$ is a perfect $k$ th power where $k=$ the denominator of the fractional root.

Fermat's last theorem [18] says, for $n>2$, the equation,

$$
x^{n}+y^{n}=z^{n}
$$

has no solution in integers, $x, y, x \neq 0$.

For 360 years mathematicians tried to resolve this conjecture but failed. Why? Since the indirect proof is not valid, one can only attempt to look for potential solution and show that every one of them does not satisfy Fermat's Equation (3). But that is like looking for a black cat in a dark room and the cat may not even be there because the search cannot be completed the potential solutions being infinite. Therefore, we look for a counterexample to it, i.e., a solution of Fermat's Equation (3). We first note that the problem is formulated in the real number system. But it has no solution there in view of the defects we have noted. Partial rectification was done in 1998 [18] which resulted in the resolution of the problem by a counterexample that proved the conjecture false and it turns out that there are countably infinite counterexamples to it [1] [18]. Full rectification of foundations is done in [1] and the rectification of the real number system is the constructivist real number system [5] an overview of which is presented in the next section.

\section{The Constructivist Real Number System}

The rectification of the real number system $\mathbf{R}$ lies in the replacement of the field axioms by three simple consistent axioms below to build the constructivist real number system $\mathbf{R}^{*}$.

\subsection{The Axioms of $R^{*}$}

Axiom 1. 0, $1 \in \mathrm{R}^{*}$.

Axiom 2. The addition table.

Axiom 3. The multiplication table.

Axiom 1 says that 0 and 1 are the additive and multiplicative identities defined by Table 1 and Table 2 .

The rest of the digits are generated by or sums of 1 . Thus, $2=1+1,3=2+$ $1,4=3+1,5=4+1,6=5+1,7=8+1$ and $9=8+1$. The base is $10=9+1$.

Both tables are extended to large numbers and small numbers using the scientific notation (metric system or base 10 numerals). Associativity, commutativity and distributivity of multiplication with respect to addition are shown by the tables and need not be taken as axioms. 
Table 1. The addition table.

\begin{tabular}{|c|c|c|c|c|c|c|c|c|c|c|}
\hline+ & 0 & 1 & 2 & 3 & 4 & 5 & 6 & 7 & 8 & 9 \\
\hline 0 & 1 & 2 & 3 & 4 & 5 & 6 & 7 & 8 & 9 & \\
\hline 1 & 2 & 3 & 4 & 5 & 6 & 7 & 8 & 9 & & \\
\hline 2 & 3 & 4 & 5 & 6 & 7 & 8 & 9 & & & \\
\hline 3 & 4 & 5 & 6 & 7 & 8 & 9 & & & & \\
\hline 4 & 5 & 6 & 7 & 8 & 9 & & & & & \\
\hline 5 & 6 & 7 & 8 & 9 & & & & & & \\
\hline 6 & 7 & 8 & 9 & & & & & & & \\
\hline 7 & 8 & 9 & & & & & & & & \\
\hline 8 & 9 & & & & & & & & & \\
\hline
\end{tabular}

Table 2. The multiplication table.

\begin{tabular}{|l|l|l|l|l|l|l|l|l|l|l|}
\hline+ & 0 & 1 & 2 & 3 & 4 & 5 & 6 & 7 & 8 & 9 \\
\hline 0 & 0 & 0 & 0 & 0 & 0 & 0 & 0 & 0 & 0 & 0 \\
\hline 1 & 0 & 1 & 2 & 3 & 4 & 5 & 6 & 7 & 8 & 9 \\
\hline 2 & 0 & 2 & 4 & 6 & 8 & & & \\
\hline 3 & 0 & 3 & 6 & 9 & & & & \\
\hline 4 & 0 & 4 & 8 & & & &
\end{tabular}

\subsection{The Inverses and Terminating Decimals}

The additive inverse of an integer $x$, denoted by $-x$, satisfies the equation,

$$
x+-x=0
$$

We write the product of integers $a$ and $b$ as $a(b)$ or $a b$. The multiplicative inverse of a nonzero integer $x$, denoted by $1 / x$, satisfies,

$$
x(1 / x)=1
$$

provided $x$ does not have a prime factor other than 2 and 5. The quotient of two integers $x$ by $y$, denoted by $x / y$ satisfies,

$$
x=y z
$$

In scientific notation we write an integer $N$ as follows:

$$
N=a_{n} a_{n-1} \cdots a_{1}=a_{n} 10^{n}+a_{n-1} 10^{n-1}+\cdots+a_{1}
$$

where the $a_{j}^{\prime}$ s, $j=1,2, \cdots, n$, are digits. A terminating decimal in the metric system or scientific notation (base 10 place-value numerals) is written as follows: 


$$
\begin{aligned}
& N . a_{n} a_{n-1} \cdots a_{1} \cdot b_{k} b_{k-1} \cdots b_{1} \\
& =a_{n} 10^{n}+a_{n-1} 10^{n-1}+\cdots+a_{1}+b_{1} / 10+b_{2} / 10^{2}+\cdots+b_{k} / 10^{k} \\
& =a_{n} 10^{n}+a_{n-1} 10^{n-1}+\cdots+a_{1}+b_{1}(0.1)+b_{2}(0.1)^{2}+\cdots+b_{k}(0.1)^{k},
\end{aligned}
$$

where $a_{n} a_{n-1} \cdots a_{1}$ is the integral part, $b_{1} b_{2} \cdots b_{k}$ the decimal part which is well-defined since 10 has only the factors 2 and 5 and its reciprocal, $1 / 10=0.1$, is a terminating decimal. Thus, a terminating decimal is defined.

What happens to $x / y$ if $x$ and $y$ are relatively prime, i.e., they have no common factor? In this case continued division does not yield a terminating decimal and therefore, the quotient is ill-defined. We extend the real number system to include not only the nonterminating decimals but also its closure in a suitable norm that we will introduce later. In $\mathbf{R}$ only division by 0 is disallowed. In $\mathbf{R}^{\star}$ division by a prime other than 2 and 5 yields a nonterminating decimal which is defined but should be disallowed in $\mathbf{R}$.

\subsection{Basic Concepts}

In scientific notation we write an integer $N$ as follows:

$$
N=a_{n} a_{n-1} \cdots a_{1}=a_{n} 10^{n}+a_{n-1} 10^{n-1}+\cdots+a_{1},
$$

where the $a_{j}$ 's, $j=1,2, \cdots, n$, are digits. A terminating decimal in the metric system or scientific notation (base 10 place-value numerals) is written as follows:

$$
\begin{aligned}
& N . a_{n} a_{n-1} \cdots a_{1} b_{k} b_{k-1} \cdots b_{1} \\
& =a_{n} 10^{n}+a_{n-1} 10^{n-1}+\cdots+a_{1}+b_{1} / 10+b_{2} / 10^{2}+\cdots+b_{k} / 10^{k} \\
& =a_{n} 10^{n}+a_{n-1} 10^{n-1}+\cdots+a_{1}+b_{1}(0.1)+b_{2}(0.1)^{2}+\cdots+b_{k}(0.1)^{k},
\end{aligned}
$$

where $a_{n} a_{n-1} \cdots a_{1}$ is the integral part, $b_{1} b_{2} \cdots b_{k}$, the decimal part which is defined since 10 has only the factors 2 and 5 and its reciprocal, $1 / 10=0.1$, is a terminating decimal. Thus, a terminating decimal is defined. A decimal is defined if all of its digits are defined or there is an algorithm for finding any digit.

\subsection{The Nonterminating Decimals}

A sequence of terminating decimals of the form,

$$
N . a_{1}, N . a_{1} a_{2}, \cdots, N . a_{1} a_{2} \cdots a_{n}, \cdots
$$

where $N$ is an integer, $a_{n}$ a digit and there is a rule for choosing each $a_{n}$, is called standard generating sequence or g-sequence. A nonterminating decimal is the g-limit (g-lim) of its nth g-term,

$$
N . a_{1} a_{2} \cdots a_{n} \cdots
$$

as $n \rightarrow \infty$, i.e., $g-\lim \left(N . a_{1} a_{2} \cdots a_{n} \cdots\right)=N . a_{1} a_{2} \cdots a_{n} \cdots$. Note that the g-limit of a nonterminating decimal is obtained by simply writing down is digits. This definition applies to terminating decimals. It also defines the natural g-norm or natural norm for $\mathbf{R}^{*}$, i.e., $\left|N . a_{1} a_{2} \cdots a_{n} \cdots\right|_{N}=N . a_{1} a_{2} \cdots a_{n} \cdots$. Thus, the g-norm of a decimal, terminating or nonterminating, is the decimal itself. The decimal in (12) is terminating when there exists an integer $k>n$ such that 


$$
a_{k}=0=a_{k+1}=a_{k+2}=\cdots
$$

For the first time (12) defines a nonterminating decimal [5]. The nth g-term of (11) approximates the nonterminating decimal (12) at maximum margin of error of $10^{-\mathrm{n}}$ provided each nth g-term is computable, i.e., there is some algorithm or rule for determining or computing the digits. The scientific calculator has this algorithm for every decimal. The g-limit of (11) is the nonterminating decimal (12) provided the nth digits are not all 0 beyond a value of $n$. In this case, we say that the g-sequence (11) converges to the nonterminating decimal (12) in the g-norm. Otherwise, it is terminating. A decimal consists of the integral part, the integer left of the decimal point; the decimal part is the sequence of digits to the right of the decimal point. The integers are ill-defined by the field axioms of the real number system but defined in the constructivist real number system by Axioms 1, 2 and 3 [5] as the integral parts of the decimals.

It would be an appropriate special problem for undergraduate mathematics major to study a class of nonterminating decimals with a given algorithm for computing its digits, e.g., normal decimal. Every digit in a normal decimal is taken at random from the ten digits $0,1,2, \cdots, 9$. To stimulate creativity, the student may think of a certain algorithm and derive the properties of the decimal generated by it. In the real number system the rationals coincide with the terminating decimals which are periodic. Since the concept "irrational" is ill-defined there are only two types of real numbers-terminating and nonterminating. The scientific calculator which has the algorithm for approximating all decimals by truncation at desired margin of error. Ramanujan's continued fraction is equivalent to a nonterminating decimal when the denominator has prime factor other than 2 or 5 .

We define the nth distance $r_{n}$ between two decimals $a, b$ as the numerical value of the difference between their nth g-terms, $a_{n}, b_{n}$, i.e., $r_{n}=\left|a_{n}-b_{n}\right|$, and their g-distance is the g-limit of $r_{n}$. The nth g-term of a nonterminating decimal repeats every preceding g-term so that if finite initial g-terms are deleted the g-terms and g-limit of the remaining g-sequence are unaltered. Thus, a nonterminating decimal has many g-sequences belonging to the equivalence class of its g-limits.

Since addition and multiplication and their inverse operations, subtraction and division, are defined only on terminating decimals computing nonterminating decimals is done by approximating each term or factor by its nth g-term (n-truncation) which is a terminating decimal and using their approximation to find the nth g-term of its sum or product. The same approximation holds for the difference and quotient (if defined). Thus, we have retained standard computation but avoided the ambiguity and contradictions of the real number system. We have also avoided vacuous approximation because nonterminating decimals are g-limits of their g-sequences which exist and belong to $\mathbf{R}^{\star}$. Moreover, we have contained the ambiguity of nonterminating decimals by approximating them by their nth g-terms. This is called truncation at the nth g-term. Approxi- 
mation makes sense only when what is being approximated is known.

As we raise $n$ in (12), the tail digits of the $n$th g-term of any decimal recedes to the right indefinitely, i.e., it becomes steadily smaller until it is indistinguishable from the tail digits of the rest of the decimals. Although it tends to 0 in the standard norm it never reaches 0 in the g-norm since the tail digits are never all equal to 0 ; it is also not a decimal since the digits are not fixed. We refer to it as an algebraic continuum distinct from the topological continuum.

At present the natural numbers are defined as 0 ; its successor, $1 ; 2$, the successor of $1 ; 3$, the successor of 2 ; etc. There is no axiom defining them. We take a surjection of the natural numbers onto the integers, $n \leftrightarrow N, N$ the integral part of a decimal and $N=0,1, \cdots$, which makes them equivalent mathematical spaces since they have exactly the same properties with respect to the binary operations + and $\times[19]$. We define a natural number as the integral part of some decimal.

The g-norm simplifies computation considerably [5] since the result is obtained digit by digit and avoids radicals which are ill-defined.

\subsection{The Dark Number $d^{*}$}

Consider the iterated product or power,

$$
(0.1)^{n}, n=1,2, \cdots ;
$$

it defines the sequence

$$
0.1,0.01,0.001, \cdots
$$

This is not a g-sequence since the g-terms do not repeat; we call it d-sequence. Each term moves towards 0 but does not reach 0 . The d-sequence defines the d-limit of the d-sequence (15) called the principal d-limit $d_{p}$ because every d-limit is derivable from it. For each $x \in \mathbf{R}^{\star}$,

$$
0<d<x
$$

Consider the sequence,

$$
(0.1)^{-n} a_{0} a_{1} \cdots a_{k}, n=1,2, \cdots ; k=0,1,2, \cdots, 9
$$

where $a_{k}$ is any of the decimals, $0,0.1,0.2,0.3, \cdots, 0.9$ and $a_{1}, \cdots, a_{k}$ decimal digits in the sequence not all 0 simultaneously. For any $x \in \mathbf{R}^{\star}, 0<d<x$. There are countably infinite $\mathrm{d}$-sequences and d-limits in (17) all of which share the same properties. The set of g-limits is not empty; we call it the dark number $d^{*}$ so that $d^{*}$ has countably infinite elements each of which satisfies,

$$
0<d_{k}<x, x \in \mathbf{R}^{*}, k=1,2, \cdots
$$

The standard limit of (17) is 0 but its d-limit $d^{*}$ is greater than 0 according to (18). Therefore, they differ by $d^{\star}$. The d-limit has special properties not shared by other elements of $\mathbf{R}^{\star}$. In fact, it has a different arithmetic.

If $x$ is a nonzero decimal, terminating or nonterminating, there is no difference between $(0.1)^{n}$ and $x(0.1)^{n}$, as $n \rightarrow \infty$, because they become indistinguishably small. This is analogous to the sandwich theorem of calculus that says, 
$\lim (x / \sin x)=1$, as $x \rightarrow 0$; in the proof, it uses the fact that $\sin x<x<\tan x$ or $1<x / \sin x<\sec x$ where both extremes tend to 1 so that the middle term tends to 1 also. In our case, if $0<x<1,0<x(0.1)^{n}<(0.1)^{n}$ and both extremes tend to 0 ; so must the middle term and they become indistinguishably small as $n \rightarrow \infty$. The middle term, however, reaches $d^{\star}$. In this sense, computation in $\mathbf{R}^{\star}$ is more precise.

If $x>1$, we simply reverse the inequality and obtain the same conclusion. Therefore, we may write, $x d_{p}=d_{p}$ (where $d_{p}$ is the principal element of $d^{*}$ ) and since the elements of $d^{*}$ share this property we may write $x d^{*}=d^{*}$, meaning, that $x d=d$ for every element $d$ of $d^{*}$. We consider $d^{*}$ the equivalence class of its elements. In the case of $x+(0.1)^{n}$ and $x$, we look at the nth g-terms of each and, as $n \rightarrow \infty, x+(0.1)^{n}$ and $x$ become indistinguishable. Now, since $(0.1)^{n}>\left((0.1)^{m}\right)^{n}>0$ and the extreme terms both tend to 0 as $n \rightarrow \infty$, so must the middle term tend to 0 so that they become indistinguishably small (the reason $d^{*}$ is called dark since it is indistinguishable from 0 but $d^{*}>0$ ).

(The dark number $d^{*}$ models the superstring mathematically. The superstring is the fundamental building block of matter. It comprises dark matter, one of the two fundamental states of matter, the other ordinary or visible matter. Dark because it is not detectable. When agitated the superstring becomes a primum, unit of visible matter, e.g., electron, +quark, -quark. They are called basic prima because they comprise every atom [20]).

In $\mathrm{R}^{*}$, the natural norm is the g-norm, i.e., if $x \in \mathrm{R}^{*}$, g-norm $(x)=x$. The nth g-term differs from $x$ by the tail end in the truncation of $x$ at the nth digit. The standard limit of the tail end differs from the g-limit by $d^{\star}$. Every mathematical space has its natural norm that facilitates computation and enhances research.

\subsection{Samples of Digital or g-Computation}

Approximation makes sense only when we know the number we are approximating and the error is bounded above by a desired number.

- Let $x=4.57628 \ldots$ The truncation (Tr) of $x$ at the $3^{\text {rd }}$ g-term is 4.576 . The distance from $x$ or error in the truncation is 0.00028 and $d^{*}<0.00028$.

- The standard distance between points $x=(2.3517 \cdots, 5.9372 \cdots)$ and $y=(1.1213 \cdots, 2.8432 \cdots)$ in the plane is approximated by the difference between their truncations at say, the 3rd g-term:

$$
\operatorname{Tr}(y)-\operatorname{Tr}(x)=3.094-1.123=1.971
$$

- We have no problem with the nth root of a perfect nth power, say, $a^{1 / 3}$ where $a=k^{2}, k$ an integer since it is defined. But how do we compute, say, $\sqrt[3]{7}$ since 7 is not a perfect square?

Step 1. Divide the interval $[0,10]$ as follows: $[0,1,2,3,4,5,6,7,8,9,10]$. Among the digits, find the largest digit whose cube does not exceed 7. Obviously, it is 1 since $1^{3}<7$ and $2^{3}>7$. Thus, the integral part of $\sqrt[3]{7}$ is 1 .

Step 2. To compute the first decimal digit divide the interval $[0.1,1]$ as follows: $[0.1,0.2,0.3,0.4,0.5,0.6,0.7,0.8,0.9,1]$. Among these decimal digits, find 
the largest digit, say, $\mathrm{b}$ such that the (1.b) ${ }^{3}$ does not exceed 7 . Clearly, it is 8 since $(1.8)^{3}=5.832<7$ and 9 does not satisfy this inequality. Thus, the closest truncation of $\sqrt[3]{7}$ at the first decimal digit is 1.8 .

Step 3. To find the next decimal digit divide the interval $[0.01,0.1]$ as follows: $[0.01,0.02,0.03,0.04,0.04,0.05,0.06,0.07,0.08,0.08,0.1]$ and find the largest digit, say, $b$ such that $(1.8 b)^{3}<7$. In case, it is 5 since $(1.85)^{3}=6.33<7$, etc.

We can repeat the calculation to find as many decimal digits as we wish in evaluating $\sqrt[3]{7}$. The scientific calculator can do this in split second because it has this algorithm for it. The computation avoids radicals which are ill-defined.

For purposes of computation we denote the nth g-term of a decimal by the functional notation $n-\xi(x)$ called n-truncation. Since a g-sequence defines or generates a decimal we call the latter its g-limit. Since nonterminating decimals cannot be added, subtracted, multiplied or divided, they must be $\mathrm{n}$-truncated first to carry out the operations on them. The margin of error at each step in the computation must be consistent (analogous to the requirement of number of significant figures in physics, the rationale being that the result of computation cannot be more accurate than any of the approximations of the terms). While we can start division by terminating decimal since it starts on the left digits the quotient does not exist when the divisor has a prime factor other than 2 or 5 .

Let $x=N \cdot a_{1} \cdots a_{n} \cdots$ and $y=M \cdot b_{1} \cdots b_{n} \cdots$, then

$$
\begin{gathered}
n-\xi(x)=N . a_{1} \cdots a_{n}, \\
n-\xi(y)=M \cdot b_{1} \cdots b_{n}, \\
n-\xi(x+y)=n-\xi(x)+n-\xi(y), \\
n-\xi(x-y)=n-\xi(x)-n-\xi(y), \\
n-\xi(x y)=(n-\xi(x))(n-\xi(y)), \\
n-\xi(x / y)=(n-\xi(x)) /(n-\xi(y)),
\end{gathered}
$$

provided $n-\xi(y) \neq 0$ as divisor. Consider the function $f\left(x_{1}, \cdots, x_{k}\right)$ of several variables; we $\mathrm{n}$-truncate $\mathrm{f}$ as follows:

$$
n-\xi\left(f\left(x_{1}, \cdots, x_{k}\right)\right)=f\left(n-\xi\left(x_{1}\right), \cdots, n-\xi\left(x_{k}\right)\right) .
$$

If $f$ is a composite function of several variables,

$$
\begin{aligned}
f( & \left.g_{1}\left(x_{1}, \cdots, x_{t}\right), \cdots, g_{s}\left(y_{1}, \cdots, y_{u}\right)\right) \text { then } \\
& n-\xi\left(f\left(g_{1}\left(x_{1}, \cdots, x_{t}\right), \cdots, g_{s}\left(y_{1}, \cdots, y_{u}\right)\right)\right) \\
& =f\left(n-\xi\left(g_{1}\left(n-\xi\left(x_{1}\right)\right), \cdots, n-\xi\left(x_{t}\right)\right), \cdots, n-\xi\left(g_{s}\left(n-\xi\left(y_{1}\right)\right), \cdots, n-\xi\left(y_{u}\right)\right)\right) .
\end{aligned}
$$

This formalizes standard computation now based on the new real numbers. The computation itself uses the g-terms of the decimals involved and provides the result directly, digit by digit; it approximates the result to within any d-term of $d^{\star}$, the closest approximation one can ever get to is $d^{\star}$. Computation using the g-norm applies to monotone increasing function since the g-terms of a decimal is monotone increasing. However, a monotone decreasing function can be converted to a monotone increasing one and g-norm computation applies to the 
latter.

\section{Theorems of $R^{*}$}

The following theorems follow from (5) and (6) of Section 3 and the ensuing discussion.

Theorem. The d-limit of the indefinitely receding to the right nth d-terms of $d^{*}$ coincides with the g-limits of the tail digits of the nonterminating decimals traced by them as the ass vary along the digits.

Corollary. The tip of the tail end of a decimal is $d^{k}$.

This means that that we cannot insert a decimal between 0 and $d^{\star}$ (the interval is full). This is true of every interval of $\mathbf{R}^{\star}$, i.e., the whole $\mathbf{R}^{\star}$ is an algebraic continuum (no gap). A decimal integer has the form N.99...; we shall prove later that they are isomorphic to the integers, i.e., the integral parts of the decimals.

Theorem. If $x$ is an integer, $x+d^{*}=x$; if $x$ is the decimal integer $N .99 \cdots, N=0,1, \cdots$ then,

$x+d^{*}=N .99 \cdots+d^{*}=N .99 \cdots+1-0.99 \cdots=N+0.99 \cdots+1-0.99 \cdots=N+1$,

$x-d^{*}=x$; if $x \neq 0, x d^{*}=d^{*}$;

$\left(d^{*}\right)^{n}=d^{*}, n=1,2, \cdots ; 1-d^{*}=0.99 \cdots ;$

$N-(N-1) .99 \cdots=1-0.99 \cdots=d^{*}$.

The equation $x+d^{*}=x$ in the theorem says that $d^{*}$ cannot be separated from an integer. Note that $x+d^{\star} \in \mathbf{R}^{\star}$. It also shows that every element of $\mathbf{R}^{\star}$ has the dark component $d^{\star}$. Recall that the g-closure of $\mathbf{R}$, i.e., its closure in the g-norm, is $\mathbf{R}^{\star}$. This is true of the additive inverses, the defined multiplicative inverses and $d^{*}$. The reciprocal of $d^{*}$ is $u^{*}$ and the reciprocal of $u^{*}$ is $d^{*}$. The the upper bounds of divergent sequences of terminating decimals and integers (a sequence is divergent if the nth terms are unbounded as $n \rightarrow \infty$, e.g., the sequence $8,88, \ldots)$. This unbounded number $u^{*}$ is countably infinite since the countable union of countable sequences is. Like $d^{*}$ it is set-valued. We follow the same convention for $u^{*}$ : whenever we have a statement " $u$ has property $\mathrm{P}$ for every element $u$ of $u^{\star}$ " we can simply say " $u^{*}$ has property P". Then $\mathrm{u}^{\star}$ satisfies these dual properties: for all $x$,

$$
x+u^{*}=u^{*} ; \text { for } x \neq 0, x u^{*}=u^{*} .
$$

Note the duality of the statements $x+d^{*}=x$ and $x d^{*}=d^{\star}$ from Equation (25) and the statements $x+u^{*}=\mathrm{u}^{*}$ and $x u^{*}=u^{*}$. Neither $d^{*}$ nor $u^{*}$ is a decimal; their properties are solely determined by their sequences. Then $d^{\star}$ and $u^{\star}$ have the following dual or reciprocal properties and relationship:

$$
0 d^{*}=0,0 / d^{*}=0,0 u^{*}=0,0 / u^{*}=0,1 / d^{*}=u^{*}, 1 / u^{*}=d^{*} .
$$

Numbers like $u^{*}-u^{*}, d^{*} / d^{*}$ and $u^{*} / u^{*}$ are still indeterminate but indeterminacy is avoided by computation with the g- or d-terms. Thus, standard g-computation applies to these non-standard elements. Moreover, we now have a defined arithmetic of infinitesimal and infinity of the traditional real number 
system and calculus where $d^{\star}$ and $u^{\star}$ are their counterparts in $\mathbf{R}^{\star}$.

The decimals are linearly ordered by the lexicographic ordering " $<$ " defined as follows: two elements of $\mathbf{R}^{\star}$ are equal if corresponding digits are equal. Let

$$
N . a_{1} a_{2} \cdots, M . b_{1} b_{2} \cdots \in \mathbf{R}
$$

Then,

$$
N . a_{1} a_{2} \cdots<M . b_{1} b_{2} \text { if } N<M \text { or if } N=M, a_{1}<b_{1} \text {; if } a_{1}=b_{1}, a<b_{2} ; \cdots,
$$

and, if $x$ is any decimal we have,

$$
0<d^{*}<x<u^{*} \text {. }
$$

The trichotomy axiom follows from the lexicographic ordering of the $\mathbf{R}^{*}$ which is linear since there is no gap. This is the natural ordering mathematicians sought among the real numbers but it does not hold in $\mathbf{R}$ because there is no linear ordering of the real numbers since $d^{*}$ is missing. This is what the counterexample to the trichotomy axiom [5] says. By inserting $d^{\star}$ in these gaps ( $d^{\star}$ lies between predecessor and successor elements of $\mathbf{R}$ ) we obtain the closure $\mathbf{R}^{*}$. Then we have the following theorem.

Theorem. The closure of $\mathbf{R}$ in the g-norm is $\mathbf{R}^{\star}$.

An open problem is whether $\mathbf{R}$ can be well-ordered by $<$. The answer is no since there are gaps in $\mathbf{R}$. However, $\mathbf{R}^{\star}$ is well-ordered.

\subsection{Decimal Integers}

Decimal integers are elements of $\mathbf{R}^{\star}$ form $N .99 \cdots, N=0,1,2, \cdots$ is called a decimal integer. We show the isomorphism between the integers and the decimal integers to justify the name in the sense of [19]. However, before doing so we first note that $1+0.99 \ldots$ is not defined in $\mathbf{R}$ since $0.99 \ldots$ is nonterminating but we can write $0.99 \cdots=1-d^{*}$ so that $1+0.99 \cdots=1+1-d^{*}=2-d^{*}=1.99 \cdots$; we now define $1+0.99 \cdots=1.99 \cdots$ or, in general, $N-d^{*}=(N-1) .99 \cdots$. Twin integers are pairs $(N,(N-1) .99 \cdots), N=1,2, \cdots$; the first and second components are isomorphic.

Let $f$ be the mapping $N \rightarrow(N-1) .99 \ldots$ and extend it to the mapping $d^{*} \rightarrow 0$ even if $d^{*}$ is not a decimal; then we show that $\mathrm{f}$ is an isomorphism between the integers and decimal integers:

$$
\begin{aligned}
f(N+M) & =(N+M-1) \cdot 99 \cdots=N+M-1+0.99 \cdots \\
& =N-1+M-1+1.99 \cdots=N-1+0.99 \cdots+M-1+0.99 \cdots \\
& =(N-1) .99 \cdots+(M-1) .99 \cdots=f(N)+f(M)
\end{aligned}
$$

Thus, addition of decimal integers is the same as addition of integers. Next, we show that multiplication is also an isomorphism.

$$
\begin{aligned}
& f(N M)=(N M-1) .999 \cdots=N M-1+0.99 \cdots \\
= & N M-N-M+1+N-1+M-1+0.99 \cdots \\
= & N M-N-M+1+(N-1) .99 \cdots+(M-1) .99 \cdots+(-1)(0.99 \cdots) \\
= & N M-N-M+1+N(0.99 \cdots)+(-1)(0.99 \cdots) \\
& +M(0.99 \cdots)+(-1)(0.99 \cdots)+0.99 \cdots
\end{aligned}
$$




$$
\begin{aligned}
& =(N-1)(M-1)+(N-1)(0.99 \cdots)+(M-1)(0.99 \cdots)+(0.99 \cdots)^{2} \\
& =(((N-1)+0.99 \cdots)((M-1)+0.99 \cdots)) \\
& =((N-1) .99 \cdots)((M-1) .99 \cdots) \\
& =(f(N))(f(M))
\end{aligned}
$$

We include in the isomorphism the map $d^{\star} \rightarrow 0$, so that its kernel is the set $\left\{d^{\star}, 1\right\}$ from which follows,

$$
\left(d^{*}\right)^{n}=d^{*} \text { and }(0.99 \cdots)^{n}=0.99 \cdots, n=1,2, \cdots
$$

If $0<x<1$, there is no difference between $\mathrm{C}$ and $x(0.1)^{n}$ since $(0.1)^{n}$ tend to $d^{*}$ in the g-norm; it follows that $x\left(d^{\star}\right)=d^{\star}$. Intuitively, this means that as the $\mathrm{d}$ and g-terms of $x(0.1)^{n}$ and $x(0.1)^{n}$ move to the right towards 0 indefinitely they become indistinguishable from each other but remain greater than zero.

Theorem. Let $x=N .99 \ldots$; then

$$
\begin{aligned}
& x+d^{*}=x, x+d^{*}=N+1, x-d^{*}=x ; \text { if } x \neq 0, x d^{*}=d^{*} \\
& \left(d^{*}\right)^{n}=d^{*}, n=1,2, \cdots, 1-0.99 \cdots=d^{*} ; N-(N-1) .99 \cdots=1.99 \cdots
\end{aligned}
$$

Note that among the nonterminating decimals, $d^{\star}$ and $0.99 \ldots$ serve as the additive and multiplicative identities, respectively. This means that $\mathbf{R}^{\star}$ has two multiplicative identity elements, namely, 1 and $0.99 \ldots$ We exhibit other properties of $0.99 \ldots$... Let $K$ be an integer, $M .99 \ldots$ and $N .99 \ldots$ decimal integers. Then

$$
\begin{aligned}
& K+M .99 \cdots=(K+M) .99 \cdots, \\
& K(M .99 \cdots)=K(M+0.99 \cdots)=K M+K(0.99 \cdots), \\
& M .99 \cdots+N .99 \cdots=(M+N+1) .99 \cdots .
\end{aligned}
$$

To verify that $2(0.999 \ldots)=1.99 \ldots$, we note that $(1.99 \ldots) / 2=0.99 \ldots$

$$
\begin{aligned}
& (M .99 \cdots)(N .99 \cdots)=(M+0.99 \cdots)(N+0.99 \cdots) \\
= & M N+M(0.99 \cdots)+N(0.99 \cdots)+(0.99 \cdots)^{2} \\
= & M N+(M-1) .999 \cdots+(N-1) .99 \cdots+0.99 \cdots \\
= & M N+(M+N-2) .99 \cdots+0.99 \cdots \\
= & M N+(M+N-1) .99 \cdots=(M N+M+N-1) .99 \cdots \\
& 0.99 \cdots+0.99 \cdots=2(0.99 \cdots)=1.99 \cdots
\end{aligned}
$$

\subsection{Adjacent Decimals and Recurring 9s}

Two decimals are adjacent if they differ by $d^{\star}$. Predecessor-successor pairs and twin integers are adjacent. In particular, $74.5700 \ldots$ and $74.5699 \ldots$ are adjacent. Since the decimals have the form $N . a_{1} a_{2} \cdots a_{n}, \cdots, N=0,1,2, \cdots$, the digits are identifiable and, in fact, countably infinite and linearly ordered by the lexicographic ordering. Therefore, they are discrete or digital and the adjacent pairs are also countably infinite. However, since their tail digits form a continuum, $\mathbf{R}^{*}$ is a continuum with the decimals its countably infinite discrete subspace.

A decimal is called recurring 9 if its tail decimal digits are all equal to 9. For 
example, $4.3299 \ldots$ and $299.99 \ldots$ are recurring 9s; so are the decimal integers. (In an isomorphism between two algebraic systems, their operations are interchangeable, i.e., they have the same algebraic structure and differ only in notation).

The recurring $9 \mathrm{~s}$ have interesting properties. For instance,

$N-(N-1) .99 \cdots=d^{*}$; such pairs are adjacent because there is no decimal between them. In the lexicographic ordering the smaller of the pair of adjacent decimals is the predecessor and the larger the successor. The average between them is the predecessor. Thus, the average between 1 and $0.99 \ldots$ is $0.99 \ldots$ since $(1.99 \ldots) / 2=0.99 \ldots$; this is true of any recurring 9 , say, $34.5799 \ldots$ whose successor is $34.5800 \ldots$ Conversely, the g-limit of the iterated or successive averages between a fixed decimal and another decimal of the same integral part is the predecessor of the former.

Since adjacent decimals differ by $d^{\star}$ and there is no decimal between them, i.e., we cannot split $d^{*}$ into nonempty disjoint sets, we have another proof that $d^{*}$ is a continuum. This is equivalent to the topological continuum. The counterexample to the trichotomy axiom debunks the idea that a terminating can be expressed as the limit of sequence of rationals since the closest it can get to it is some rational interval containing rationals whose relationship to it is unknown.

The g-sequence of a nonterminating decimal reaches its g-limit, digit by digit. This is one of the advantages of computation in the g-norm. Moreover, a nonterminating decimal is an infinite series of its digits:

$$
N . a_{1} a_{2} \cdots a_{n} \cdots=N+0 . a_{1}+0.0 a_{2}+\cdots+0.00 \cdots 0 a_{n}+\cdots ; 0.99 \cdots
$$

\section{5. $\mathbf{R}^{*}$ and Its Subspaces}

We add the following results to the information we now have about the various subspaces of $\mathbf{R}^{\star}$ to provide a full picture of the structure $\mathbf{R}^{\star}$. The next theorem is a definitive result about the continuum $\mathbf{R}^{\star}$.

Theorem. In the lexicographic ordering $\mathbf{R}^{\star}$ consists of adjacent predecessor-successor pairs (each joined by $\mathrm{d}^{\star}$ ); hence, the g-closure $\mathbf{R}^{\star}$ of $\mathbf{R}$ is a continuum.

Proof. For each $N, N=0,1, \cdots$, consider the set of decimals with integral part $N$. Take any decimal in the set, say, $N . a_{1} a_{2} \cdots$, and another decimal in it. Without loss of generality, let $N . a_{1} a_{2} \cdots$ be fixed and let it be the larger decimal. We take the average of the nth g-terms of $N . a_{1} a_{2} \cdots$ and the second decimal; then take the average of the nth g-terms of this average and $N . a_{1} a_{2} \cdots$; continue. We obtain the d-sequence with nth d-term, $(0.5)^{-n} a_{1} a_{2} \cdots a_{n+k}$, which is a $\mathrm{d}$-sequence of $d^{\star}$. Therefore, the g-limit of this sequence of averages is the predecessor of $N . a_{1} a_{2} \cdots$ and we have proved that this g-limit and $N . a_{1} a_{2} \cdots$ are predecessor-successor pair, differ by $d^{*}$ and forms a continuum. Since the choice of $N . a_{1} a_{2} \cdots$ is arbitrary then by taking the union of these predecessor-successor pairs of decimals in $\mathbf{R}^{\star}$ (each joined by the continuum $d^{\star}$ ) for all integral parts $N, \quad N=0,1, \cdots$, we establish that $\mathbf{R}^{\star}$ is a continuum.

However, the decimals form countably infinite discrete subspace of $\mathbf{R}^{\star}$ since 
there is a scheme for labeling them by integers and the integers are discrete and countably infinite. We can imagine them as forming a right triangle with one edge horizontal and the vertical one extending without bounds. The integral parts are lined up on the vertical edge and joined together by their branching digits between the hypotenuse and the horizontal that extend to $d^{\star}$ which is adjacent to 0 (i.e., differs from 0 by a dark number) at the vertex of the horizontal edge.

Corollary. $\mathbf{R}^{*}$ is non-Archimedean but Hausdorff in both the standard and the g-norm and the subspace $\mathbf{R}$ of decimals is countably infinite, hence, discrete but Archimedean and Hausdorff. Clearly, $\mathbf{R}$ or the set of decimals is a subspace of the constructivist real number system $\mathbf{R}^{\star}$.

The following theorem is true in $\mathbf{R}$ (standard norm). Therefore, we do not bring in $d^{*}$ in the proof so that this is really a theorem about the decimals in the standard norm but not so in the g-norm where the decimals merge into a continuum at their tail digits and cannot be separated.

Theorem. Every real number is isolated from the rest [20].

Proof. Let $p \in \mathbf{R}$ be a nonterminating decimal and $\left\{q_{n}\right\}$ a sequence of rationals converging to $\mathrm{p}$ from the left. Let $d_{n}$ be the distance from $q_{n}$ to $p$ and take an open ball of radius $d_{n} / 10^{n}$, with center at $q_{n}$. Note that $q_{n}$ tends to $\mathrm{p}$ but distinct from it for any $n$. Take an open ball of radius $d_{n} / 10^{n}$, centered at $\mathrm{p}$ and take the union of open balls, centered at $q_{n}$, as $n \rightarrow \infty$ and call it $U$. If $\mathrm{r}$ is any decimal to the left of $p$, then $r$ is separated from $p$ by at least $d^{\star}$ which is isolated from the rest of the real numbers. The same result holds for any $r$ distinct from and to the right of $p$.

Here is another surprise that contradicts a theorem in the real number system.

Theorem. The rationals and nonterminating decimals are separated, i.e., they are not dense in their union (the first indication of discreteness of the decimals [21]).

Proof. Let $p \in \mathbf{R}^{\star}$ be a nonterminating decimal, and let $q_{n}, n=1,2, \cdots$ be a sequence of rationals towards and left of $p$, i.e., $n>m$ implies $q_{n}>q_{m}$; let $r_{n}$ be the distance from $q_{n}$ to $p$ and take an open ball of radius $r_{n} / 10^{n}$, center at $q_{n}$. Note that $q_{n}$ tends to $p$ but distinct from it for any $n$. Let $U=\bigcup U_{n}$, as $n \rightarrow \infty$, then $U$ is open and if $q$ is any real number, rational or nonterminating to the left of $p$ then $q$ is separated from $p$ by disjoint open balls, one in $U$, center at $q$ and the other in the complement of $U$, center at $p$. Since the rationals are countable union of open sets $U$ for all the rationals, the nonterminating decimal $p$ is separated from all the rationals.

We use the same argument if $p$ were rational and since the reals has countable basis we take $q_{n}$ a nonterminating decimal, for each $\mathrm{n}$, at center of open ball of radius $d_{n} / 10^{n}$. Take $U$ to be the union of such open balls. Using the same argument, a real number in $U$, rational or nonterminating, is separated by disjoint open balls centered at $p$.

Thus, every decimal is separated from the rest of $\mathbf{R}$, the terminating decimals 
from the nonterminating decimals and from each other. Clearly, the last two theorems do not hold in $\mathbf{R}^{\star}$. We state a theorem that is true in $\mathbf{R}^{\star}$.

Theorem. The largest and smallest elements of the open interval $(0,1)$ are $0.99 \ldots$ and $d^{*}$, respectively [21].

Proof. Let $C_{n}$ be the nth term of the g-sequence of $0.99 \ldots$ For each $n$, let $I_{n}$ be open segment (segment that excludes its endpoints) of radius $10^{-2 n}$ centered at $C_{n}$. Since $C_{n}$ lies in $I_{n}$ for each $n, C_{n}$ lies in $(0,1)$ as $n$ increases indefinitely. Therefore, the decimal $0.99 \ldots$ lies in the open interval $(0,1)$ and never reaches 1. To prove that $0.99 \ldots$ is the largest decimal in the open interval $(0,1)$ let $x$ be any point in $(0,1)$. Then $x$ is less than 1 . Since $C_{n}$ is strictly increasing n can be chosen so that $x$ is less than $C_{n}$ and this is so for all subsequent values of $\mathrm{n}$. Therefore, $x$ is less than $0.99 \ldots$ and since $x$ is any decimal in the open interval $(0,1)$ then $0.99 \ldots$ is, indeed, the largest decimal in the interval and is itself less than 1.

To prove that $1-0.99 \ldots$ is the smallest element of $\mathbf{R}$, we note that the g-sequence of $1-0.99 \ldots$ is steadily decreasing. Let $K_{n}$ be the nth term of its g-sequence. For each $n$, let $B_{n}$ be an open interval with radius $10^{-2 n}$ centered at $k_{n}$. Then $K_{n}$ lies in $B_{n}$ for each $\mathrm{n}$ and all the $B_{n}$ s lie in the open set in $(0,1)$. If $y$ is any point of $(0,1)$, then $y$ is greater than 0 and since the generating sequence $1-$ $0.99 \ldots$ is steadily decreasing $n$ can be chosen large enough such that $y>K_{n}$ and this is so for all subsequent values of $n$. Therefore, $y>1-0.99 \ldots$ and since the choice of $y$ is arbitrary, $1-0.99 \ldots$ is the smallest number in the open interval $(0,1)$ greater than 0 .

This is not true in $\mathbf{R}^{\star}$ since $1-0.99 \ldots$ and $0.99 \ldots$ are not defined. Most textbook in college algebra have these errors.

Goldbach's Conjecture. An even number greater than 2 is the sum of two primes.

The original proof of this 250-year-old conjecture is in [20] but we reproduce it here for completeness. Like Fermat's equation the conjecture is indeterminate and need not have a solution in $\mathbf{R}$. It has, however, solutions in $\mathbf{R}^{\star}$.

We note first that an integer is a prime if it leaves a positive remainder when divided by another integer other than 1 . We retain this definition of a prime in $\mathbf{R}^{\star}$ but the remainder is $d^{*}$ which is not a real number.

Proof. The conjecture is not vacuous since it is true when $p=q=2$. In fact, it is true when $n<10$. Let $n$ be an even number greater than 10 . Then there is some prime number $\mathrm{p}$ greater than 3 and another number $q$ such that $p+q=n$. If $q$ is prime then the theorem is proved; otherwise, it must be divisible by some integer ( 0 remainder) other than 1 and $q$. Then we add to $q$ the dark number $d^{*}>0$ and we have $q+d^{*}=q$ and division of $q$ by any nonzero integer yields nonzero remainder $d^{\star}$, i.e., $q$ is prime.

It follows from the proof that every element of $\mathbf{R}^{\star}$ greater than 3 is a prime. In $\mathbf{R}$ with all its defects, every real number is composite since if $x \in \mathbf{R}, x=\sqrt{x} \sqrt{x}$, where $\sqrt{x}$ is some decimal. In this sense $\mathbf{R}$ and $\mathbf{R}^{*}$ are dual. For example, $\sqrt[4]{3}=(\sqrt{3})(\sqrt{3})$. Of, course, this is still true with the corrections except that it 
can be approximated only when the number under the operator $\sqrt[4]{ }$ is not a perfect fourth power.

\subsection{The Structure of $\mathrm{R}^{*}$}

We now have a sense of how the decimals are arranged by the lexicographic ordering. Below is a sequence of successor-predecessor pairs at the boundaries of a rational and nonterminating decimals.

$$
\begin{aligned}
& N .4999100 \cdots \\
& N .4998999 \ldots \\
& N .4999999 \ldots \\
& N .499999899 \ldots
\end{aligned}
$$

The ellipses after the first and second rows are filled with $d^{\star}$ alone. The ellipses between the third and fourth rows are filled with $d^{*}$ and nonterminating decimals. The largest decimal in the open interval $(N .49100, N .49999899 . .$.$) is$ the decimal integer $N .49100 \ldots$ and the smallest is the nonterminating decimal $N .49999899 .$. Starting from the bottom going up, the decimals with integral part $N$ are arranged as predecessor-successor pairs each joined by $d^{\star}$. Each gap (represented by ellipses) is filled by countably infinite adjacent predecessor-successor pairs also joined by $d^{\star}$ so that their union is a continuum. Clearly, $\mathbf{R}^{\star}$ is linearly ordered by $<$, the lexicographic ordering; $d^{\star}$ joins every decimal to its successor and $d^{*}$ cannot be split from either.

\subsection{Resolution of a Paradox and Other Important Results}

Every convergent sequence has a g-subsequence defining a decimal adjacent to its standard limit [5].

If a decimal is terminating it is the standard limit itself. We express this as a theorem:

Theorem. The difference between the standard limit and the g-limit is $d^{*}$.

(2) It follows from (1) that the standard limit of a sequence of terminating decimals can be found by evaluating the g-limit of its g-subsequence which is adjacent to it. This is an alternative way of computing the limit of ordinary sequence.

(3) In [22] several counterexamples to the generalized Jourdan curve theorem for $n$-sphere are shown where a continuous curve has points in both the interior and exterior of the $n$-sphere, $n=2,3, \cdots$, without crossing the n-sphere. Our explanation is: the functions cross the n-sphere at dark numbers.

(4) Given two decimals and their g-sequences and respective nth g-terms $A_{n}$, $B_{n}$ we define the nth g-distance as the g-norm $\left|A_{n}-B_{n}\right|$ of the difference between their nth g-terms. Their g-distance is the $\mathrm{g}$ - $\lim \left|A_{n}-B_{n}\right|$, as $n \rightarrow \infty$, which is adjacent to the standard norm of the difference. Advantage: the g-distance is the g-norm of their decimal difference; the difference between nonterminating decimals cannot be evaluated otherwise. Moreover, this notion of distance can be extended to n-space, $n>2,3, \cdots$, and the distance between two points can be 
evaluated digit by digit in terms of their components without the need for evaluating roots. In fact, any computation in the g-norm yields the results directly, digit by digit, without the need for intermediate computation such as evaluation of roots in standard computation. (The decimals are "glued" together by $\mathrm{d}^{*}$ to form the continuum $\mathbf{R}^{*}$ )

(5) We know from [1] that the celebrated Banach-Tarski paradox is vacuous, does not exist.

(6) Well-ordering Theorem. Every sequence $R^{\star}$ bounded below has a greatest lower bound and every sequence in $\mathbf{R}^{\star}$ bounded above has a least upper bound (the bound in either case is a decimal, terminating or nonterminating).

Proof. If the sequence has a lower bound it has standard limit $S$. Then $S+d^{*}=$ $S$ is the greatest lower bound because any number to the right of $S$ will not be a lower bound. The proof is similar for the least upper bound.

The proof of this theorem in $\mathbf{R}$ is flawed because it uses a variant of the axiom of choice. It also involves vacuous concepts.

(7) If the standard limit of a sequence of decimals exists then it is constructible since it is adjacent to the g-limit of some g-sequence.

We have clearly identified the ambiguous concepts of $\mathbf{R}$, defined some of them in $\mathbf{R}^{\star}$ and discarded some that cannot be fixed, e.g., the irrationals. We have also identified certain theorems in $\mathbf{R}$ that are false in $\mathbf{R}^{\star}$. All theorems in real analysis that relies on the axiom of choice are not true, e.g. the Heine-Borel theorem and the existence of non-measurable set [13].

\subsection{Advantages of the G-Norm}

- Avoids indeterminate forms.

- In computation, the g-norm yields the answer directly, digit by digit, and avoids intermediate computation.

- Since the standard limit is adjacent to the g-limit of some g-sequence, evaluating it reduces to finding some nonterminating decimal adjacent to it. A decimal is approximated by the appropriate nth g-term (n-truncation).

- Calculation of distance between two decimals with the g-norm is direct, digit by digit, and involves no root.

- In computation, taking root of a prime is avoided by using $\mathrm{n}$-truncation; the error $<10^{-n}$.

- The g-norm is the natural norm for $\mathbf{R}^{\star}$. It simplifies computation and provides the best approximation.

- Most of all, while the standard norm brings in the nonexistent radicals the g-norm does not. Thus, the g-norm is the natural norm for $\mathbf{R}^{\star}$.

Every mathematical space has its natural norm that simplifies computation and enhances discovery. Gadgets for computation are designed based on the natural norm. For example, in computation the scientific calculator gives the result in decimal. This means that the calculator works in such a way that the digits are given in the metric system (scientific notation). In other words, the cal- 
culator has the algorithm for computing the g-sequence of a decimal and, naturally, the g-limit as well. This is true of the computer. The algorithm defines an exponential function directly and without a flaw. Extension to fractional exponent is easy since the calculator approximates the g-limits of roots.

We conclude this section with the resolution of Fermat's last theorem.

\section{The Counterexamples to FLT}

Given the contradiction in negative statement, we use Fermat's equation in place of Fermat's last theorem; its solutions are counterexamples to FLT. We summarize the properties of the digit 9.

1) A finite string of $9 \mathrm{~s}$ differs from its nearest power of 10 by 1 , e.g., $10^{100}-99 \cdots 9=1$.

2) If $N$ is an integer, then $(0.99 \cdots)^{N}=0.99 \cdots$ and, naturally, both sides of this equation have the same g-sequence. Therefore, for any integer $\mathrm{N}$, $((0.99 \cdots) 10)^{N}=(9.99 \cdots) 10^{N}$.

3) $\left(d^{*}\right)^{N}=d^{*} ;((0.99 \cdots) 10)^{N}+d^{*}=10^{N}, N=1,2, \cdots$

Then the exact solutions of Fermat's equation are given by the triples $(x, y, z)=\left((0.99 \cdots) 10^{T}, d^{*}, 10^{T}\right), T=1,2, \cdots$, that clearly satisfies Fermat's equation,

$$
x^{n}+y^{n}=z^{n},
$$

for $n=N T>2$. Moreover, for $k=1,2, \cdots$, the triples $(k x, k y, k z)$ also satisfy Fermat's equation. They are the countably infinite counterexamples to FLT that prove the conjecture false. This is the original resolution of FLT in [18]. (One counterexample is, of course, sufficient to disprove the conjecture)

\section{Transcendental Functions}

Algebraic functions are defined only when their domains are terminating decimals. Those that are undefined we include them under transcendental functions where the argument ranges through $\mathbf{R}^{\star}$. There are special functions which do not presently belong to the class of transcendental functions. They will be treated in a sequel part of under the mathematics of the grand unified theory [23].

If $y=f(x)$ is a transcendental function we define $f(x)=\mathrm{g}-\lim (f(s))$ as $s \rightarrow$ $x$. The limiting process is taken along a g-sequence. There are, of course, functions which do not have g-sequences. They are special functions which require a different treatment. This will be discussed in a different paper. Consider the exponential function.

Let $x=N \cdot x_{1} x_{2} \cdots$, then $\mathrm{e}^{x}=\mathrm{e}^{N \cdot x_{1} x_{2} \cdots}$; if we replace $x$ by $y=f(x)$ then,

$$
\mathrm{e}^{f(x)}=\mathrm{e}^{\mathrm{g}-\lim (f(s))} \text { as } s \rightarrow x .
$$

It follows from (2) that

$$
\log _{\mathrm{e}} f(x)=\log _{\mathrm{e}}(\mathrm{g}-\lim f(s)) \text {, as } s \rightarrow x .
$$

The approach to exponential and logarithmic functions base 10 is the same: 


$$
10^{f(x)}=10^{\mathrm{g}-\lim (f(s))} \text { as } s \rightarrow x .
$$

Let $\log f(x)$ be a logarithmic function base 10 . Then we have this definition:

$$
\log f(x)=\log (g-\lim f(s)), \text { as } s \rightarrow x .
$$

Equations (2)-(5) define the exponential and logarithmic functions for the first time (without flaw). They are inverse functions of each other. The circular functions and their inverses are treated in the same way.

Since the exponential and logarithmic functions base e are convertible to exponential and logarithmic functions base 10, we have now defined transcendental functions.

We can approximate sums and products of these functions at a given point by suitable truncations. For example:

- To compute $\mathrm{e}^{x}$ at $x=a$, we calculate the $\operatorname{g-limit}(f(x))$, as $x \rightarrow a$; $\mathrm{e}^{x}$ is approximated by suitable truncation.

- To evaluate $\pi$ up to the $5^{\text {th }}$ decimal digit, we use the infinite series expansion of $\pi$ which is $\pi=3.14159 \ldots$ Then we truncate it at the $5^{\text {th }}$ digit and the error will be less than $(0.1)^{5}$. The standard decimal expansion of $\pi$ is the closest approximation of its g-limit and differs from the g-limit by $d^{\star}$. Of course, the scientific calculator can calculate $\pi$ quickly by its algorithm.

- We illustrate the algorithm for calculating the nth root of a decimal using $\sqrt{3}$.

Step 1. The largest integer $\mathrm{n}$ such that such that $n^{2}<3$ is 1 . Therefore, the integral part of $\sqrt{3}$ is 1 .

Step 2. Divide the interval $[0,1]$ into subintervals at the following points: 0 , $0.1,0.2,0.3,0.4,0.5,0.5,0.6,0.7,0.8,0.9,1$. The largest division point $\mathrm{n}$ such that $(1 . n)^{2}<3$ is $n=0.7$. Therefore, the truncation of $\sqrt{3}$ at the first decimal digit is 1.7 .

We continue the calculation and find that $\sqrt{3}=1.732050808 \cdots$. This is exactly how the scientific calculator computes nth roots. Then calculation with fractional exponents, terminating or nonterminating, is known. The only requirements for valid approximation is: what is being approximated is known and the error is bounded away from 0 by $d^{\star}$.

Theorem. The decimals are ordered by the lexicographic ordering in $\mathbf{R}^{\star}$ and the trichotomy axiom is true in $\mathbf{R}^{\star}$ and follows from the lexicographic ordering.

Proof. The closure of $\mathbf{R}$ in the g-norm which is $\mathbf{R}^{\star}$ fills in the gaps in $\mathbf{R}$ with $\mathrm{d}^{*}$ that allows the linear ordering of $\mathbf{R}^{*}$ by the lexicographic ordering.

Theorem. Let $K$ be an integer, $M .99 \ldots$ and $N .99 \ldots$ decimal integers. Then

$$
\begin{gathered}
K+M .99 \cdots=K+M+0.99 \cdots=(K+M) .99 \cdots, \\
K(M .99 \cdots)=K(M+0.99 \cdots)=K M+K(0.99 \cdots)=K M+K \\
(\text { since } K(0.99 \cdots)=K), \\
M .99 \cdots+N .99 \cdots=(M+N+1) .99 \cdots
\end{gathered}
$$

Theorem. In the lexicographic ordering $\mathbf{R}^{*}$ consists of adjacent predeces- 
sor-successor pairs (each joined by $d^{\star}$ ); therefore, the g-closure $\mathbf{R}^{*}$ of $\mathbf{R}$ is a continuum.

Corollary. The average between the predecessor and its successor is the successor.

Corollary. $\mathbf{R}^{*}$ is non-Archimedean but Hausdorff in both the standard and the g-norm and the subspace $\mathbf{R}$ of decimals is countably infinite, hence, discrete but Archimedean and Hausdorff.

Theorem. $\mathbf{R}^{\star}$ is a continuous linear array of predecessor-successor pairs with $d^{*}$ inserted between them.

\section{The Complex Vector Plane}

The complex vector plane $\mathbf{C}^{\star}$ is the rectification of the complex number system C. It the circular functions as a subspace.

\subsection{The Element $\mathrm{j}$ as Operator on Plane Vectors}

The rectification of $\mathbf{C}$ involves the replacement of the vacuous concept $\mathrm{i}$ by the left-right plane vector operator $\mathbf{j}$, rotation of the clockwise or positive rotation $(x, 0)$ about the origin by $\pi / 2$. Then the coordinate axes are generated by applying $\mathbf{j}$ on the $x$-axis, i.e., $\mathbf{j}(x, 0)=\mathbf{j} y$ (rotation of the $x$-axis by $\pi / 2$ ), applying $\mathbf{j}$ on the $\mathrm{j} y$-axis, i.e., $\mathrm{jj}(\mathrm{y}, 0)=(-x, 0)$ (rotation of the $-x$-axis by $\pi / 2)$ to obtain the negative $x$-axis, and $\mathrm{j}$ on the $-x$-axis, i.e., $\mathrm{jjj}(-x, 0)=\mathrm{j}(-y, 0)$ (rotation of the negative $\mathrm{x}$-axis by $\pi / 2$ ) to obtain the $-y$-axis and applying $\mathrm{j}$ on the negative $y$-axis, i.e. $\mathrm{jjjj}(-x, 0)=(x, 0)$ (rotation of the negative $y$-axis by $\pi / 2)$ to obtain the $x$-axis. Thus, we have the cyclic values of the composites of $\mathbf{j}$ with itself:

$$
\mathbf{j}(\mathbf{1})=\mathbf{j}, \mathbf{j} \mathbf{j}(\mathbf{1})=\mathbf{j}^{2}(\mathbf{1})=-\mathbf{j}, \mathbf{j j}^{2}(\mathbf{1})=-\mathbf{j}^{3}(\mathbf{1})=-\mathbf{j}, \mathbf{j} \mathbf{j}^{3}(\mathbf{1})=\mathbf{j}^{4}(\mathbf{1})=\mathbf{1} \text {. }
$$

The complex vector plane is generated by applying $\mathbf{j}$ on $(x, 0)$ through $\theta, 0 \leq \theta$ $\leq 2 \pi$. We define the operator $-\mathbf{j}$ as inverse operator of $\mathbf{j}$, i.e., when applied on a vector $\mathbf{v}$ we rotate it clockwise by $\pi / 2$ so that $-\mathbf{j}(\mathbf{1})=\mathbf{j}(-\mathbf{1})$. Applying composite mappings on the unit vector 1 along the $\mathrm{x}$-axis successively, we have the four cyclic images of 1 in (1) in reverse order. For $n>4$, the cycle is repeated and we obtain $\mathbf{j}^{n}=\mathbf{j}\left(\mathfrak{j}^{\mathrm{n}-1}\right), n=1,2, \cdots$, where we define $\mathbf{j}^{0}=\mathbf{1}$.

\subsection{Scalar and Vector Operations}

For completeness, we introduce scalar multiplication. If $\alpha$ is an element of $\mathbf{R}^{\star}$ (scalar), $\alpha \mathrm{j}$ is a vector of modulus $\alpha$ along the y-axis. Note that scalar multiplication commutes with $\mathbf{j}$. If $\beta$ is another scalar,

$$
(\alpha \beta) \mathbf{j}=\mathbf{j}(\alpha \beta)=(\mathbf{j} \alpha) \beta=\beta(\mathbf{j} \alpha)=(\beta \alpha) \mathbf{j},
$$

which follows from $\mathbf{j}$ being left-right operator and the commutativity of multiplication in $\mathbf{R}^{\star}$. From commutativity and associativity of multiplication we have, for $\alpha, \beta, \gamma \in \mathbf{R}^{*}$,

$$
(\alpha \beta \gamma) \mathbf{j}=\alpha(\beta \gamma) \mathbf{j}=(\alpha \beta)(\gamma \mathbf{j})=(\beta \alpha) \gamma \mathbf{j}=(\gamma \beta \alpha) \mathbf{j}=\mathbf{j}(\gamma \beta \alpha) .
$$


Also, from distributivity of multiplication in $\mathrm{R}^{\star}$ with respect to addition we have,

$$
\alpha(\beta+\gamma) \mathbf{j}=(\alpha \beta+\alpha \gamma) \mathbf{j}=(\alpha \beta) \mathbf{j}+(\alpha \gamma) \mathbf{j}
$$

Thus, we have retrieved the basic properties of the complex plane. We call $\mathbf{j}$ the complex vector operator. Every vector in the complex vector number system has new real and complex components; conversely, a vector is the vector sum of its new real and complex components. Thus, a vector $\mathbf{z}$ in it has standard form,

$$
\mathbf{z}=\alpha+\beta \mathbf{j} \text { or } \mathbf{z}=(\alpha, \beta \mathbf{j}), \alpha, \beta \in \mathbf{R}^{*} .
$$

The arithmetic of the complex plane holds provided that whenever 1 appears as a factor we interpret it as a unitary vector operator so that $1 \alpha=\alpha$, the vector of modulus 1 along the $\mathrm{x}$-axis. Thus, we retain in the complex vector plane and the vector algebra of the complex plane, the latter isomorphic to the former. All concepts of the complex plane except $\mathbf{i}$ which is replaced by $\mathbf{j}$ carry over to the complex vector plane. For example, the norm or modulus of the complex vector $\mathbf{v}=\alpha+\beta \mathbf{j}$, denoted by $|\mathrm{v}|$, is given by,

$$
|\mathbf{v}|=\left(\alpha^{2}+\beta^{2}\right)^{1 / 2}
$$

the square root of the product of $\mathbf{z}$ and its conjugate, $\alpha-\beta \mathbf{j}$. The dot product of vectors $\mathbf{u}$ and $\mathbf{v}$ is given by

$$
\mathbf{u} \cdot \mathbf{v}=|\mathbf{u}||\mathbf{v}| \cos \theta \text { if } \mathbf{u} \neq \mathbf{0}, \mathbf{v} \neq \mathbf{0}, \mathbf{u} \cdot \mathbf{v}=0 \text { if } \mathbf{u}=\mathbf{0} \text { or } \mathbf{v}=\mathbf{0},
$$

where $\theta \in \mathbf{R}^{*}$.

Two parallel vectors with the same norm are equivalent. Therefore, a vector can be translated to a standard vector with initial point at the origin.

The vector additive and multiplicative identities are the vectors 0 and 1 , respectively, where the latter called the unit vector coincides with its real component 1 . The scalars are subject to the operations in $\mathbf{R}^{\star}$ and scalar multiplication on vectors which are both commutative and associative. With the complex vector arithmetic now defined we have verified that the operator $\mathbf{j}$ applies to any vector in the complex vector plane. Applying $\mathbf{j}$ on the vector $\mathbf{z}$ of (5), we have,

$$
\mathbf{j}(\mathbf{z})=\mathbf{j}(\alpha+\mathbf{j} \beta)=-\beta+\mathbf{j} \alpha,
$$

a positive rotation of vector $\mathbf{z}$ by $\pi / 2$.

\subsection{The Operator $\mathrm{h}_{\theta}$}

We introduce the more general complex left-right operator $\mathrm{h}_{\theta}$ on the complex vector plane appropriate for analytical work:

$$
\mathbf{h}_{\theta}(a+\mathbf{j} b)=\mathbf{r} \mathrm{e}^{\mathbf{j} \theta}=\mathbf{r}(\cos \theta+\mathbf{j} \sin \theta)
$$

where $a, b \in \mathbf{R}^{\star}, \mathbf{r}$ is the radius vector of $\mathbf{r} \mathrm{e}^{\mathrm{j} \theta}$ represented by an arrow with initial point at the origin forming an angle $\varphi$ with the $\mathrm{x}$-axis and using the calculator algorithm for square root $|\mathbf{r}|=\sqrt{a^{2}+b^{2}}$ is the modulus, a decimal, terminating 
or nonterminating; $\mathrm{e}^{\mathrm{j} \theta}$ is the unit circle with center at the terminal point of $\mathbf{r}$ and forming an angle $\varphi$ with the $\mathrm{x}$-axis, $0 \leq \varphi \leq 2 \pi, 0 \leq \theta \leq 2 \pi$. Clearly, $\mathrm{re}^{\mathrm{j} \theta}$ covers the entire complex vector plane.

The unit circle $\mathrm{e}^{\mathrm{j} \theta}$ is the well defined counterpart of $\mathrm{e}^{\mathrm{i} \theta}$. If we vary $\alpha$ and $\beta$ along $\mathbf{R}^{\star}$ and $\theta$ in $[0,2 \pi]$ the terminal point of $\mathbf{z}$ covers the entire complex vector plane. Geometrically, $r$ varies in $[0, \infty)$ and rotates around the origin from 0 to $2 \pi$ as the unit circle with center at the terminal point of $\alpha+\mathbf{j} \beta$ rotates from $\theta=0$ to $\theta=2 \pi$. Then a point $z_{0}$ is given by

$$
\mathbf{z}_{0}=\mathbf{h}_{\theta}\left(\alpha_{0}+\mathbf{j} \beta_{0}\right)=\mathrm{r}_{0}\left(\cos \theta_{0}+\mathbf{j} \sin \theta_{0}\right), \theta_{0} \in[0,2 \pi] .
$$

If $r_{0}=1,(1)$ reduces to the equation of the unit circle with center at the origin. This operator applied on a vector along the $\mathrm{x}$-axis rotates it by $\theta$, reducing to operator $\mathbf{j}$ when $\theta=\pi / 2$.

In the solution of the gravitational n-body problem [24], the operator that generates the spiral covering of a vortex is a variant of $\mathbf{h}_{\theta}$ and has the form,

$$
\mathbf{z}=a \mathrm{e}^{\lambda t} \eta(t)
$$

where $\eta$ is given by the expression,

$$
\mathbf{h}_{\lambda}(t)=\cos \lambda+\mathbf{j} \mathrm{e}^{-t} \sin \lambda,
$$

depending on the specific cases and phases of the evolving boundary conditions of the problem; here $\lambda$ is the constant of integration in the solution of the constraint equation of the associated optimal control formulation of this problem [25].

Suppose vector $\mathbf{z}$ has initial and terminal points $(\alpha, \mathbf{j} \beta)$ and $(\gamma, \mathbf{j} \zeta)$, respectively. Then,

$$
\mathbf{z}=\mathbf{z}_{1}-\mathbf{z}_{2}=(\alpha, \mathbf{j} \beta)-(\gamma, \mathbf{j} \zeta)
$$

Therefore,

$\mathbf{j}(\mathbf{z})=\mathbf{j}\left(\mathbf{z}_{1}-\mathbf{z}_{2}\right)=\mathbf{j}((\alpha+\mathbf{j} \gamma \beta)-(\gamma+\mathbf{j} \zeta))=\mathbf{j}(\alpha-\gamma)+(\zeta-\beta)=(\zeta-\beta)+\mathbf{j}(\alpha-\gamma)$,

is a counterclockwise rotation of $\mathbf{z}$ by $\pi / 2$. In general, a polygon of $\mathrm{n}$ edges $\mathbf{e}_{1}, \cdots, \mathbf{e}_{n}$ may be represented as the vector sum $\mathbf{e}_{1}+\cdots+\mathbf{e}_{n}$ or its resultant $\mathbf{r}$. Then $\mathbf{j}\left(\mathbf{e}_{1}+\cdots+\mathbf{e}_{n}\right)=\mathbf{j}(\mathbf{r})$ is a counterclockwise rotation of the polygon by $\pi / 2$.

The operator $\mathbf{j}$ is an automorphism of the complex vector plane. Its additive inverse $-j$ is clockwise rotation about the origin by $\pi / 2$. There is, however, a new vector operation in the complex vector plane that may not have counterpart in other vector spaces: the product of two vectors. Let $\mathbf{u}=\alpha+\mathbf{j} \beta, \mathbf{v}=\gamma+\mathbf{j} \zeta$. Their product is given by

$$
\mathbf{u v}=(\alpha+\mathbf{j} \beta)(\gamma+\mathbf{j} \zeta)=(\alpha \gamma-\beta \zeta)+\mathbf{j}(\alpha \zeta+\beta \gamma)
$$

which, restricted to the complex plane, reduces to standard complex vector multiplication with $\mathbf{j}$ replaced by $\mathrm{i}$. This is a particularity of the complex vector plane 
not shared by other vectors spaces. Consider vectors,

$$
\mathbf{z}_{1}=(\alpha+\mathbf{j} \beta), \mathbf{z}_{2}=(\gamma+\mathbf{j} \zeta)
$$

then,

$$
\mathbf{z}_{1} \mathbf{z}_{2}=\mathbf{h}_{\theta}(\alpha+\mathbf{j} \beta) \mathbf{h}_{\phi}(\gamma+\mathbf{j} \zeta)=\mathbf{r}_{1} \mathbf{r}_{2}(\cos (\theta+\varphi)+\mathbf{j} \sin (\theta+\varphi)),
$$

where $r_{1}, r_{2}$ are the respective moduli of the vectors of $\mathbf{z}_{1}, \mathbf{z}_{2}$ and $\theta, \phi$ their arguments. Note that this product of complex vectors is distinct from both the dot and vector products in a vector space. It is an extension of multiplication of complex numbers. Since the product of two complex vectors is a complex vector the product vector can be extended to any number of factors.

The additive inverse of a complex vector is quite obvious. For the multiplicative inverse we reduce its reciprocal to standard form. For instance, if $\mathbf{z}=\alpha+\mathbf{j} \beta$ then its multiplicative inverse $\mathbf{z}^{-1}$ is given by

$$
\mathbf{z}^{-1}=1 /(\alpha+\mathbf{j} \beta)=(\alpha-\mathrm{j} \beta) /\left(a^{2}+b^{2}\right)=\mathbf{h}\left(\mathbf{z}^{-1}\right)=\mathbf{j}(1 / r)(\cos \theta-\mathbf{j} \sin \theta)
$$

where $1 / r=\bmod \left(\mathrm{z}^{-1}\right)$ and $\theta=\arg \left(\mathrm{z}^{-1}\right)$. Then division of complex vector by another reduces to its multiplication by the inverse of the other. In general, if $\mathbf{z}_{1}=r_{1}(\cos \theta+\mathbf{j} \sin \theta), \quad \mathbf{z}_{2}=r_{2}(\cos \varphi+\mathbf{j} \sin \varphi)$, then

$\mathbf{z}_{1} / \mathbf{z}_{2}=\left(r_{1} / r_{2}\right)(\cos \theta-\sin \phi)$. Note that the operator $\mathbf{h}_{\theta}$ is really equivalent to the old notation $\mathrm{e}^{\mathrm{i} \theta}$ and the latter may be used in place of $\mathbf{h}_{\theta}$ when convenient.

Again, the operator $\mathbf{j}$ played a crucial role in solving the gravitational $n$-body problem [26] by generating the spiral covering of the underlying vortex by the gravitational flux streamlines as solutions of the conjugate equations obtained by the integrated Pontrjagin maximum principle [24] from the optimal control formulation of this problem. The $\mathrm{n}$ bodies and their rotating trajectories were obtained along specific spiral streamlines by the fractal-reverse-fractal algorithm [25] using a body at the core of the cosmological vortex as fractal generator. Elliptical orbit in the underlying spinning vortex is attained when the gravitational flux pressure balances the centrifugal force, its ellipticity being due to radial fluctuation of this balance by virtue of the oscillation universality principle [27], another expression of perfect balance being unstable which accounts for the fact that orbits of cosmological bodies are elliptical.

The extension to $\mathrm{n}$-space using the orthogonal unit vectors $\mathbf{e}_{1}, \cdots, \mathbf{e}_{n}$ as bases is quite obvious. This section establishes the complex vector plane as extension of $\mathbf{R}^{*}$.

\section{Introduction to Discrete Calculus}

With the g-norm we set up the mechanism for discrete differentiation and integration since both involve limits. We introduce some concepts of discrete differentiation and integration. Discrete the domain is $\mathbf{R}^{\star}$.

To find the derivative at $x=s$, where $\mathrm{s}$ is terminating decimal, we find the nth g-term of $\Delta f \Delta x$, as $\Delta x \rightarrow 0$, where $x \rightarrow s, \Delta x=x-s$ and $\Delta x \neq 0$. This is simply done by steady division to generate the g-sequence of the derivative and its 
g-limit. Then for computation purposes we only need its truncation. We take values of the g-terms at successive points $x_{0}, x_{1}$, etc., and $\Delta_{0}=x_{0}-s, \Delta x_{1}=x_{1}-s$, etc. The g-term is either increasing or decreasing and truncated from above or from below accordingly. If the derivative of $f(x)$ exists in the sense of calculus it also exists in discrete calculus and the differentiation rules of calculus applies. We have here a simpler technique for optimization. Moreover, even function having no derivative in calculus such as set-valued function or function having set-valued derivative may have an optimum. Ref. [26] discuss set-valued functions and derivatives including wild oscillation of the form $f(x)=\sin ^{n}\left(1 / x^{2}\right), n=1,2, \cdots$. When $\Delta x \rightarrow 0$ in the standard norm but $\Delta \mathrm{f}$ tends to a nonzero decimal then $\Delta f \Delta x$ diverges.

The extension of our computational technique to composite function of several functions of several variables is straight forward and similar to the techniques of traditional calculus. The only innovation here is the use of truncation for finding g-sequences. The graphs of well-behaved functions in $R^{*} \times R^{*}$ and $R \times$ $\mathbf{R}$ are the same since every missing element is a dark number squeezed between adjacent decimals which is not detectable. The functions of discrete calculus are defined on the decimals. They are discrete-valued. N-space is now constructed as $\mathbf{R}_{1}^{*} \times \mathbf{R}_{2}^{*} \times \cdots \times \mathbf{R}_{n}^{*}, n=1,2, \cdots, N$. Incidentally the Lorentz transformation reduces the space of the theory of relativity to 3 -space. That is why it leads to strange consequences.

Consider the function $y=f(x)$ in the interval $[a, b]$ and subdivide $[a, b]$ by the finite set of points (decimals) $\left\{x_{k}\right\}, k=1,2, \cdots, s$, where $a=x_{1}, b=x_{s}$. We further subdivide the set by the finite set of points $\left\{x_{m}\right\}, m=1,2, \cdots, t$, take $\left\{x_{k}\right\} \cup\left\{x_{m}\right\}=\left\{x_{n}\right\}, n=1, \cdots, w=s+t$, and call it $\left\{x_{n}\right\}, n=1,2, \cdots, w$, where we relabel the points of the union of the two sets, preserving their lexicographic ordering and taking $a=x_{1}, b=x_{t}$, a refinement of both $\left\{x_{k}\right\}$ and $\left\{x_{m}\right\}$. For a well-behaved function, except at points where it is undefined, there is no significant difference for purposes of evaluating its value or limit in taking midpoints of the subsegments determined by each refinement of the subdivision of the interval $[a, b]$.

One advantage of discrete function is: we need not differentiate it to find a maximum or minimum; naturally, problems of optimization unsolvable in calculus may be solved here. This technique applies to some functions with set-valued derivatives such as the schizoid or curve with cusp. It is also applicable to set-valued functions. We call an ordinary function well behaved function. An example of a function that is not well behaved is the wild oscillation $f(x)=\sin ^{n} 1 / x, n=1,2, \cdots, k$. Such function is raw material for building the generalized integral and derivative [28].

Consider the arc of the function $y=f(x)$ over the interval $[a, b]$ and let the set of points $\left\{x_{k}\right\}, k=1,2, \cdots, s$, subdivide the interval $[a, b]$ suitably so that each local maximum or minimum is isolated in some interval. This is possible since the values of $f(x)$ over the decimals are countably infinite and discrete. Unless 
the maximum or minimum lies at an end point, its neighborhood will contain interval on which $f(x)$ is increasing on one side and decreasing on the other. At the same time, the end point of a function in an interval is either minimum or maximum. In fact, every closed interval in the range of a function contains its maximum or minimum. Without loss of generality, consider interval $[c, d]$ containing a maximum. Subdivide the interval $[c, d]$ and take successive refinements $\left\{x_{m}\right\}$ of $\left\{x_{k}\right\}\left(\left\{x_{m}\right\}\right.$ relabeled suitably) until the values of $f\left(X_{m}\right)$, tapers to a constant $\alpha$ along $\left\{x_{m}\right\}, m=1,2, \cdots, w$. In this new setting the values of a function, being discrete, is no different from a sequence of numbers. As the values become close to each other they contain a d-sequence with a set of digits in its terms receding to the right and forming a d-sequence of $d^{\star}$ and another set of values that remain fixed. The latter defines a decimal, a local maximum in this interval. By suitable translation of the function the minimum can be similarly obtained (the end point is either a maximum or minimum). Then the absolute maximum of $f(x)$ is the maximum of $M_{k}, k=1,2, \cdots, m$. In this algorithm for finding the $\mathrm{g}$-limit of a function there will be, in general, several inequivalent g-sequences each a g-sequence of a local maximum. A single g-sequence may split into distinct g-sequences in further computation of the nth g-terms when their limits are close to each other. Some functions have countably infinite maxima, e.g., infinitesimal zigzag and wild oscillation [24] [27].

By suitable linear transformation the minimum can be found in a similar way. This approach is both intuitive and computational. A more sophisticated version of it for discrete function is approximation of continuous function by a polygonal line developed in [29] [30].

We extend our method to the calculation of the length of an arc of a curve. Consider the function $y=f(x)$ over the interval $[a, b]$. Let the set of points $\left\{x_{k}\right\}, k=1,2, \cdots, s$, subdivide the interval $[a, b]$ and form the sum,

$$
L_{k}=\sum\left(x_{k+1}-x_{k}\right)^{2}+\left(f\left(x_{k+1}-x_{k}\right)^{2}\right)^{1 / 2}, k=1,2, \cdots, s
$$

where $a=x_{1}, b=x_{s}$. Take refinements of $\left\{x_{m}\right\}$ of $\left\{x_{k}\right\}$ ( $\left\{x_{m}\right\}$ relabeled suitably) until the values of $L_{m}$ tapers to a constant $\Gamma$; we call $\Gamma$ the length of the curve of $y=$ $f(x)$ over $[\mathrm{a}, \mathrm{b}]$.

In calculus the right derivative of a curve at point $P$ is obtained by drawing a line from $P$ to a point $Q$ nearby and moving $Q$ towards $P$ from the right along the curve, the derivative being the limit of the slope of the line $P Q$ as $Q$ moves towards $P$ (actually, $Q$ moves along discrete points, i.e., approximation of "continuous" curve by polygonal line). We find the right discrete derivative similarly by taking $Q$ to move along discrete set of points, the advantage being that this is discrete computation. The right discrete derivative is found in the same manner by taking the limit of the quotient $\Delta f(x) / \Delta x$, as $\Delta x \rightarrow 0^{+}$, along suitable refinement. When the right and left derivatives at a point are equal then we say that the curve is discretely regular there. To find the left discrete derivative of the 
function $y=f(x)$ at the point $P(b, f(b)]$ on the interval $[a, b]$, we assume $f(x)>0$ and increasing. Subdivide the interval $[a, b]$ by the set of points $\left\{x_{k}\right\}, k=1,2, \cdots, s$, where $a=x_{1}, b=x_{s}$, and form the quotient,

$$
D_{L}(f(b))=\left(f\left(x_{k+1}-x_{k}\right)\right) /\left(x_{k+1}-x_{k}\right) \text {. }
$$

We take successive refinements of $\left\{x_{k}\right\}$ to obtain the g-sequence of a decimal. Then $D_{L}$ is either terminating, nonterminating or zero taking approximation in each case. The right derivative $D_{R}$ can be computed similarly. When the function is discretely regular at $x$ we denote its discrete derivative at $x$ by $D_{x}$. Here we can find the left minimum and right minimum which need not be equal. The same is true of the left maximum and right maximum, left minimum and right maximum and left maximum, right minimum. Thus, the optimization applies to functions that have no optimum in the traditional sense including non-smooth functions.

We introduce the notion of locally approximating the shape of a monotone increasing function on $[a, b]$ at a point by its derivative at $P$, as $P$ traces the arc over $[a, b]$ obtained by finding the limit of the minimum of the maximum horizontal distance between the derivative function and the function itself as $P$ traces the arc [27]. Computation is straight forward for well-behaved functions. The limit gives the shape of the curve in a small neighborhood of $P$ expressed by the following theorem for smooth curves that applies as well to discrete curves since the gaps are dark.

The Minimax Principle. When the minimum of the maximum horizontal distance between two simple smooth arcs with no inflection point can be made arbitrarily small then an element of arc and variation of derivative at a point on one approximates the other [27].

We define the integral of $f(x)$ over the interval $[a, b]$ as the limit of the sums of the areas of the trapezoidal areas under the curve through successive refinements determined by the midpoints of the subsegments at each refinement as we do in calculus except that it is simpler here since the upper and lower sums coincide. This is another advantage with discrete function. The indefinite integral of $f(x)$ is simply the area under $f(x)$ over the interval $[a, x]$. Note that the integral of $f(x)$ is independent of the derivative. To evaluate it we divide the interval $[a, b]$ into subintervals by the points $\left\{x_{k}\right\}, k=1,2, \cdots, s, a=x_{1}, b=x_{s}$, form the sum,

$$
\sum_{[a, b]} f(x)=\sum\left(x_{k+1}-x_{k}\right)\left(f\left(x_{k+1}\right)+f\left(x_{k}\right)\right) / 2,
$$

from $k=1$ to $k=s$, and find its limit through successive refinements of $\left\{x_{m}\right\}$, as $m \rightarrow \infty$.

From these examples, we find that computation in the standard norm reduces to finding the nonterminating decimal adjacent to the result and, therefore, approximable to any desired margin of error.

This is just a framework for building discrete calculus appropriate for computing and simulation. Simulation is important for finding ballpark estimate of 
hidden forces (attractive or repulsive). For example, distortion of the orbit of a planet reveals the presence of some cosmological body whose gravity impinges on the planetary orbit.

\section{Conclusions}

It would appear that the rectification of $\mathbf{R}$ only offered mathematical peace of mind. There is a universe of difference between $\mathbf{R}$ and $\mathbf{R}^{\star}$. For example, the 360-year-old Fermat's last theorem [18] has no solution in $\mathbf{R}$ since $\mathbf{R}$ is inconsistent. However, $\mathbf{R}^{\star}$ has countably infinite counterexamples to it proving that FLT is false [18]. We list some of the major achievements.

- The 250-year-old Goldbach's conjecture [31] has no proof in $\mathbf{R}$ for the same reason but it has a proof in $\mathbf{R}^{*}$.

- Many fields of traditional mathematics, aside from R, e.g., number theory, set theory and symbolic logic, are dead or almost dead because there is practically no research going on and, naturally, there is paucity of publications in those fields. They share most of the defects of $\mathbf{R}$ and its foundations and the fields of their applications application inherit their defects. For example, since mathematics is the language of science, traditional physics, especially, quantum physics, is near the point of stagnation according to Lee Smolin [32]. This is due to the fact that most of modern physics, particularly, quantum physics, is still framed by Einstein's theory of relativity.

- The theory is at the point of extinction. In fact, it is difficult to find a paper on relativity during the last 100 years beyond the early work of Einstein and Hawking.

- Consequently, physicists are still looking for the fundamental building block of matter at CERN [33] unaware of the fact that it was discovered in 1997 and was the key to the solution of the gravitational n-body problem.

- It was the discovery of the superstring that launched the development of the grand unified theory (GUT) which was completed in 2008 [23].

- Discovery of an error and its rectification becomes a milestone in the development of a field. We have seen this in mathematics. In physics, the weakness of Einstein's special and general theory of relativity, particularly, the lack of adequate method and, in general relativity, ambiguity of physical concepts such as black hole and curvature of space pushed them towards extinction.

- The introduction of qualitative mathematics and modelling [34] led to the development of GUT. Its development was proposed by Einstein to unify gravity with the weak and strong forces of quantum physics through GUT but it fell through because the theory is anchored on the wrong premise that there was no absolute frame of reference for our universe. There is-dark matter.

- The discovery of the stable and indestructible dark matter debunks Relativity's premise of absence of matter in space and returns the ether, which was vanished by Einstein's theory of relativity, with a new name-the super- 
string - fundamental building block of matter [23].

- The theoretical and practical applications of GUT range through computer science [2] [3] [4], physics [16] [23] [35]-[41], astrophysics [23] [25] [26] [28] [36] [40] [42] [43] [44] [45], cosmology [45] [46], neuroscience [15] [47] [48], genetics [49] [50], biology [51] [52], global geology and oceanography [53] [54] [55], atmospheric and climatic sciences [53] [54] [56], engineering [57], development sciences [57] [58] and medicine [49] [50] [59] [60]. All told over 70 papers and books have been published in these fields by the author since the application of qualitative mathematics and modeling to sole the gravitational n-body problem n 1997.

- The most significant achievement of qualitative mathematics is the resolution of Hilbert's 23 problems in mathematics [61]. The solution of Problem 6 is GUT [23].

- L C. Young did not simply extend the methods of the calculus of variations required by Problem 23, he introduced the generalized curves and surfaces and a new norm now called the Young Measure [24] [62] [63] that he applied to functional analysis and optimal control theory in $\mathrm{R}^{n}, n \geq 3$, and solved the calculus of variations problem using constructivist method.

The good news here is that every new field that opens up in the rectification yields a robust and fertile field for research. Analysis alone has hundreds of active fields. $\mathbf{R}^{\star}$ and the complex vector field are only an introduction to real and complex analysis. Complex analysis is an endangered species but turning it into a constructivist mathematical space, rewriting the correctportion, discovering new theorems and sorting and discarding erroneous ones would require graduate students in the tens of thousands.

Finally, one might ask: With all those defects, how come we have built such giant and indispensable discipline as mathematics? The reason: mathematicians are smart enough to induce its offspring-approximation, perturbation and optimization to remedy or contain its defects so that they do not restrain the development of mathematics and science.

Note. What used to be called dynamic modeling in the references is now qualitative modeling.

\section{Acknowledgements}

Improvement in this manuscript is due to insightful comment by one of the reviewers. A good reviewer plays a significant role in the advancement of mathematics by raising appropriate questions and important issues in the field. Some reviewers even recommend references to improve the manuscript.

\section{References}

[1] Escultura, E.E. (2015) The Resolution of the Great $20^{\text {th }}$ Century Debate in the Foundations of Mathematics. Advances in Pure Mathematics, 6, 144-158.

http://file.scirp.org/Html/3-5301056_63915.htm 
https://doi.org/10.4236/apm.2016.63012

[2] Escultura, E.E. (2009) The New Real Number System and Discrete Computation Calculus. Neural, Parallel and Scientific Computation, 17, 59-84.

[3] Escultura, E.E., Bhaskar, T.G., Leela, S. and Laksmikantham, V. (2009) Revisiting the Hybrid Real Number System. Nonlinear Analysis, C-Series. Hybrid Systems, 3, 101-107. https://doi.org/10.1016/j.nahs.2008.11.001

[4] Escultura, E.E. (2009) Extending the Reach of Computation. Applied Mathematics Letters, 21, 1074-1081. https://doi.org/10.1016/j.aml.2007.10.027

[5] Escultura, E.E. (2016) The Constructivist Real Number System. Advances in Pure Mathematics, 6, 597-607. http://www.scirp.org/journal/apm/

[6] Andrew David Irvine, Bertrand Russell; andrew.irvine@ubc.ca.

[7] Intuitionism in the Philosophy of Mathematics. https://plato.stanford.edu/entries/intuitionism/

[8] Zach, R. (2005) Hilbert's Program Then and Now. arXiv:math/0508572 [math.LO]

[9] Russell's Letter to Frege. http://www.logicmuseum.com/reference/fregerussell1904.htm

[10] Weisstein, E.W. Russell's Antinomy. MathWorld. http://mathworld.wolfram.com/RussellsAntinomy.html

[11] Weisstein, E.W. Brouwer Fixed Point Theorem. MathWorld. http://mathworld.wolfram.com/BrouwerFixedPointTheorem.html

[12] Weisstein, E.W. Zermelo-Fraenkel Set Theory. MathWorld. http://mathworld.wolfram.com/Zermelo-FraenkelSetTheory.html

[13] Royden, A. (1983) Real Analysis. 3rd Edition, MacMillan, New York.

[14] Bell, J.L. The Axiom of Choice. https://plato.stanford.edu/entries/axiom-choice/

[15] Escultura, E.E. (2015) Rational Thought, Cognition and Knowledge. International Education Research, 3, 21-37. http://www.todayscience.org/IER/article/ier.v3i1p21.pdf https://doi.org/10.12735/ier.v3i1p21

[16] Escultura, E.E. (2007) The Pillars of the New Physics and Some Updates. Nonlinear Studies, 14, 241-260.

[17] Young, L.C. (1980) Mathematicians and Their Times. North-Holland, Amsterdam.

[18] Escultura, E.E. (1998) Exact Solutions of Fermat's Equation (Definitive Resolution of Fermat's Last Theorem. Nonlinear Studies, 5, 227-254.

[19] Itô, K. (1993) Encyclopedic Dictionary of Mathematics. 2nd Edition, Corporate Mathematical Society of Japan, MIT Press, Cambridge, MA.

[20] Escultura, E.E. (2003) The New Mathematics and Physics. Applied Mathematics and Computation, 138, 127-149. https://doi.org/10.1016/S0096-3003(02)00126-1

[21] Escultura, E.E. (2002) The Mathematics of the New Physics. Applied Mathematics and Computations, 130, 45-169. https://doi.org/10.1016/S0096-3003(01)00088-1

[22] Horgan, H. (1993) The Death of Proof. Scientific American, 269, 92-103. https://doi.org/10.1038/scientificamerican1093-92

[23] Escultura, E.E. (2008) The Grand Unified Theory. Nonlinear Analysis: A-Series: Theory, Methods and Applications, 69, 823-831.

[24] Young, L.C. (1969) Lectures on the Calculus of Variations and Optimal Control Theory. W. B. Saunders, Philadelphia. 
[25] Escultura, E.E. (2001) Vortex Interactions. Problems of Nonlinear Analysis in Engineering Systems, 7, 30-44.

[26] Escultura, E.E. (1997) The Solution of the Gravitational n-Body Problem. Nonlinear Analysis, Series Ai Theory, Methods and Applications, 30, 5021-5032. https://doi.org/10.1016/S0362-546X(96)00133-2

[27] Zeigler, B.P. (2005) An Introduction to Calculus Course Based on DEVS: Implications of a Discrete Reformulation of Mathematical Continuity. International Conference on Simulation in Education ICSiE05, New Orleans, 23-25 January 2005.

[28] Escultura, E.E. (1993) Diophantus: Introduction to Natural Philosophy (With Counterexamples to Fermat's Last Theorem. Kalikasan Press, Manila.

[29] Zeigler, B.P. (2004) Continuity and Change (Activity) Are Fundamentally Related in DEVS Simulation of Continuous Systems. Keynote Talk at AI, Simulation and Planning AIS 04, Kor, 4-6 October 2004.

[30] Escultura, E.E. (2111) Scientific Natural Philosophy. Bentham Science Publishing (Ebook). http://www.eurekaselect.com/53770/volume/1

[31] Davies, P.J. and Hersch, R. (1981) The Mathematical Experience. Birkhäuser, Boston.

[32] Smolin, L. (2008) The Trouble with Physics. Penguin Books, New York.

[33] CERN. https://en.wikipedia.org/wiki/CERN

[34] Escultura, E.E. (2013) The Logic and Fundamental Concepts of the Grand Unified Theory. Journal of Modern Physics, 4, 213-222.

http://file.scirp.org/Html/21-7501396_36280.htm https://doi.org/10.4236/jmp.2013.48A021

[35] Escultura, E.E. (2007) Dynamic Modeling and the New Mathematics and Physics. Neural, Parallel and Scientific Computations, 15, 527-538.

[36] Escultura, E.E. (2000) Set-Valued Differential Equations and Applications to Quantum Gravity. Mathematical Research, 6, 58-69.

[37] Escultura, E.E. (2009) Qualitative Model of the Atom, Its Components and Origin in the Early Universe. Nonlinear Analysis: Real World Applications, 11, 29-38. https://doi.org/10.1016/j.nonrwa.2008.10.035

[38] Escultura, E.E. (2013) Chaos, Turbulence and Fractal: Theory and Applications. International Journal of Modern Nonlinear Theory and Application, 2, 176-185. https://doi.org/10.4236/ijmnta.2013.23025

[39] Escultura, E.E. (2001) From Macro to Quantum Gravity. Problems of Nonlinear Analysis in Engineering Systems, 7, 56-78.

[40] Escultura, E.E. (2010) The Leap from Traditional to the New Science. Nonlinear Studies, 21, 283-291.

[41] Escultura, E.E. (2009) The Mathematics of the Grand Unified Theory. Nonlinear Analysis, A-Series. Theory, Methods, 71, e420-e431. https://doi.org/10.1016/j.na.2008.11.003

[42] Escultura, E.E. (1999) Superstring Loop Dynamics and Applications to Astronomy and Biology. Nonlinear Analysis, A-Series: Theory, Methods and Applications, 35, 259-268.

[43] Escultura, E.E. (2005) Dynamic Modeling of Chaos and Turbulence. Nonlinear Analysis, A-Series. Theory, Methods and Applications, 63, e519-e532.

https://doi.org/10.1016/j.na.2005.02.052

[44] Escultura, E.E. (1999) Recent Verification and Applications. 2rd International Con- 
ference Tools for Mathematical Modeling, St. Petersburg, 4, 74-89.

[45] Escultura, E.E. (2010) Our Fractal Universe and Applications. International Conference on Dynamic Systems and Applications, Morehouse College, Atlanta.

[46] Escultura, E.E. (2012) The Big Bang and What It Was. In: O'Connell, J.R. and Hale, A.L., Eds., The Big Bang. Theory, Assumptions and Problems, Nova Science Publishers, 61-102.

[47] Escultura, E.E. (2012) The Physics of Intelligence. Journal of Education and Learning, 1, 51-64.

http://www.ccsenet.org/journal/index.php/jel/article/view/20095/13268 https://doi.org/10.5539/jel.v1n2p51

[48] Escultura, E.E. (2014) The Physics of the Mind. The Journal of the Science of Healing Outcome, 6, 6-15.

[49] Escultura, E.E. (2015) Genetic Alteration, Modification and Sterilization with Applications to the Treatment of Genetic Diseases. The Journal of the Science of Healing Outcomes, 7, 30-45.

[50] Escultura, E.E. (2017) The Gene as Natural Nanomaterial for Treatment of Diseases. Journal of Biophysics, 7, 25-40.

http://file.scirp.org/Html/3-1850143_73254.htmhttp://file.scirp.org/Html/3-185014 3_73254.htm

[51] Escultura, E.E. (2015) The Unified Theory of Evolution. Journal of the Science of Healing Outcomes, 8, 5-18.

[52] Escultura, E.E. (2010) The Origin and Evolution of Biological Species. The Journal of the Science of Healing Outcomes, 6-7, 17-27.

[53] Escultura, E.E. (2001) Turbulence: Theory, Verification and Applications. Nonlinear Analysis, Series A: Theory, Methods and Applications, 47, 5955-5966.

[54] Escultura, E.E. (2001) Chaos, Turbulence and Fractal. Indian Journal of Pure and Applied Mathematics, 32, 1539-1551.

[55] Escultura, E.E. (2013) Problems of Modelling in Global Geology and Oceanography, Problems of Nonlinear Analysis in Engineering Systems. International Federation of Nonlinear Analysts-Russian Academy of Natural Sciences, 19, 164-178.

[56] Escultura, E.E. (2014) Is Human Activity Linked to Climate Change? Atmospheric and Climate Sciences, 4, 305-316. http://file.scirp.org/Html/15-4700251_45173.htm.

[57] Escultura, E.E. (2018) The Grand Unified Theory with Technological Applications, Journal of modern Physics. [In Press]

[58] Escultura, E.E. (2011) Qualitative Modeling for Complex Systems, Problems of Nonlinear Analysis in Engineering Systems. International IFNA-ANS Scientific Journal, 2, 79-85.

[59] Escultura, E.E. (2012) Electromagnetic Treatment of Genetic Diseases. Biomaterials and Nanobiotechnology, 3, 292-300. http://file.scirp.org/Html/7-3200175_18989.htm https://doi.org/10.4236/jbnb.2012.322036

[60] Escultura, E.E. (2015) Extending Electromagnetic Treatment to Infectious Diseases including the Ebola Virus. Journal of Biomaterials and Nanobiotechnology, 6, 100-115. http://file.scirp.org/Html/3-3200379_55595.htm https://doi.org/10.4236/jbnb.2015.62010

[61] Weisstein, E.W. Hilbert's Problems, MathWorld. http://mathworldwolfram.com/HilbertsProblems.html 
[62] Young, L.C. (1937) Generalized Curves and the Existence of an Attained Absolute Minimum in the Calculus of Variations. Comptes Rendus de la Société des Sciences et des Lettres de Varsovie, 30, 211-234.

[63] Young, L.C. (1955) On Generalized Surfaces of Finite Topological Types. Memoirs of the AMS, Philadelphia. 